\title{
Modules over Infinite Dimensional Algebras
}

\author{
A dissertation presented to \\ the faculty of \\ the College of Arts and Sciences of Ohio University
}

\author{
In partial fulfillment \\ of the requirements for the degree \\ Doctor of Philosophy
}

Lulwah Al-Essa

May 2015

(C) 2015 Lulwah Al-Essa. All Rights Reserved. 
This dissertation titled

Modules over Infinite Dimensional Algebras

by

\section{LULWAH AL-ESSA}

has been approved for

the Department of Mathematics

and the College of Arts and Sciences by

Sergio López-Permouth

Professor of Mathematics

Robert Frank

Dean, College of Arts and Sciences 


\begin{abstract}
AL-ESSA, LULWAH, Ph.D., May 2015, Mathematics

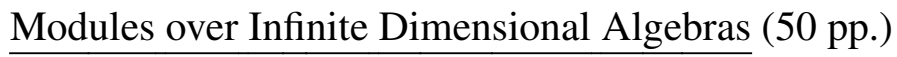

Director of Dissertation: Sergio López-Permouth

Let $\mathrm{A}$ be an infinite dimensional $K$-algebra, where $K$ is a field and let $B$ be a basis for $A$. In this Dissertation, we explore a property of the basis B that guarantees that $K^{B}$ (the direct product of copies indexed by $B$ of the field $K$ ) can be made into an $A$-module in a natural way. We call bases satisfying that property "amenable," and we show that not all amenable bases yield isomorphic $A$-modules. Then we consider a relation (which we name congeniality) that guarantees that two different bases yield isomorphic $A$-module structures on $K^{B}$. We present several examples in the familiar setting of the algebra $K[x]$ of polynomials with coefficients in $K$ and the Laurent Polynomial Algebra $K\left[x, x^{-1}\right]$. Finally, we introduce some results regarding these notions in the context of Leavitt Path Algebras.
\end{abstract}


To My Parents, Prof. AlJawharah Bubshait and Dr. Mohammed Al-Essa

To my Brothers Eng.Omar, Anas and Mouathe

To my Lovely Little sister Sara

This wouldn't have been possible without you. 


\section{ACKnowledgments}

First and foremost, I would like to express my deepest gratitude to Allah The All mighty (God) The Most Gracious, The Most Merciful, for all His giving and grace and for providing me the blessings while I was completing this dissertation.

I also would like to express my gratitude and special thanks to my advisor, Dr. López-Permouth, for his support, patience, and motivation through all stages of my research. He always has been available to advise me and provide me with guidance, wide experience and knowledge to successfully accomplish both my coursework and this project. I am very grateful for all the help he provided me throughout my graduate studies. Thanks so much for all what you have done.

Besides my advisor, I would like to acknowledge the individuals who agreed to be members of my committee. Dr. Dinh Van Huynh, Dr. Alexei Davydov, and Dr. Jeffrey Dill who dedicated much of there time and provided great guidance. I also want to thank them for making my defense a pleasurable moment.

I would like to extend my sincerest thanks and appreciation to my amazing role model, my mother AlJawharah Bubshait. She believes that if you educate a boy you educate a person but if you educate a girl you educate a family. Her unconditional faith has been a great inspiration in completion of this thesis. She has been helpful in providing me great advices and useful tips throughout my experience. I am forever grateful for her infinite love and endless support. After Allah's blessings, I never would have been able to achieve my goals without her guidance and encouragement. I would also like to extend my deepest gratitude to my father, Mohammed Al-Essa, for his massive contribution of time through this entire journey. He often said that education is the key to success in life. He's always been there for me and pushing me to be the best person I can be. I am grateful for his guidance and motivation to be able to complete this work. Mom and Dad, I am proud to have you both in my life; your love and sacrifices brought me where I am 
today. Thank you for believing in me. I truly appreciate everything that you have done; I can't thank you enough for that.

I would like to take this opportunity to express my deepest gratitude to my brothers Omar, Anas, Mouathe, and my lovely little sister Sara for their everlasting love and constant encouragement. They have been with me in every step on my life, and their kindness, patience and support make me grateful to be their sister. Despite the geographical distance, my family was always nearby; my aunts, my uncles, my cousins and my sister in law deserve my heartfelt thanks and appreciation.

Finally, further acknowledgement and thanks are due to all my friends especially Elham, Maha, Lamees, Najat, Heba and Alanoud for their attention, advice, and support through this entire process. 


\section{Table of Contents}



Dedication ......................... 4

Acknowledgments ....................... 5

1 Introduction and Preliminaries $\ldots \ldots \ldots \ldots \ldots$

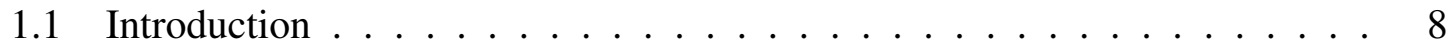

1.2 Preliminaries . . . . . . . . . . . . . . . . 9

1.3 Matrix Representations of Linear Mappings on $K^{(\omega)} \ldots \ldots \ldots \ldots \ldots$

2 Related Topics . . . . . . . . . . . . . . . . . . . . . . . . . 17

2.1 Formal Calculus and Vertex Operator Algebra . . . . . . . . . . . 17

2.2 Semigroup Rings and Group Rings . . . . . . . . . . . . . . . . . . . 19

2.3 Hamel Bases . . . . . . . . . . . . . . . . . . . . . 21

3 The Central Notion . . . . . . . . . . . . . . . . . 23

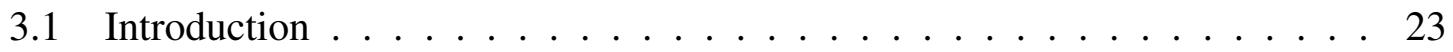

3.2 Modules Constructed from Amenable Bases . . . . . . . . . . . . . 24

4 Isomorphic Modules . . . . . . . . . . . . . . . . . . . . . . 28

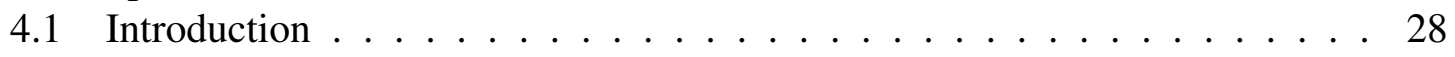

4.2 Isomorphic Modules Based on Different Bases . . . . . . . . . . . . 31

5 Leavitt Path Algebra $\ldots \ldots \ldots \ldots$. . . . . . . . . . . . 40

6 Future Work . . . . . . . . . . . . . . . . . . . . . . . . 48

Bibliography . . . . . . . . . . . . . . . . . . . 49 


\section{Introduction and Preliminaries}

\subsection{Introduction}

Let $A$ be an algebra over a field $K$ and let $\mathcal{B}$ be a basis. Then $A=\bigoplus_{b \in \mathcal{B}} K b=K^{(\mathcal{B})}$ is the direct sum of copies of the field $K$ indexed by $\mathcal{B}$. This is because the elements of $A$ are finite linear combinations of elements of the base. One can think of those linear combinations as being full, possibly infinite, linear combinations of elements of $\mathcal{B}$ with the restriction that only finitely many coefficients are allowed to be non-zero. Thusly, associated with every algebra $A$ there is another $K$-vector space, the product $P=\prod_{b \in \mathcal{B}} K b=K^{\mathcal{B}}$ of copies of $K$ (namely, the set of all infinite linear combinations of elements of $\mathcal{B}$, without any restriction on coefficients). A natural question is whether $P$ has a module structure over the algebra $A$ that is naturally induced by the knowledge of the products of elements from the basis. The answer to the question is that while all the various bases produce versions of $P$ that are isomorphic to one another as vector spaces, some versions will be amenable to be made into $A$-modules and some others will not. For that reason, we introduce here the notion of an amenable basis basically being one for which the leading question has a positive answer. We use the notation $P=\mathcal{M}_{\mathcal{B}}$ to refer to the module structure on $\mathrm{P}$ for an amenable basis $\mathcal{B}$.

A follow up question is whether amenable bases yield isomorphic module structures. One approach to attain a sufficient condition is through the notion of congeniality of bases. A basis $\mathcal{B}$ is congenial to a basis $C$ if for every element $c \in C$ there exits only finitely many elements $b \in \mathcal{B}$, when represented in terms of $C$ use a non-zero coefficient in their representation of $c$ in terms of $\mathcal{B}$. It turns out that the congeniality relation is not an equivalence relation; in fact, it is not symmetric. We provide examples of bases $\mathcal{B}$ and $\mathcal{C}$ such that $\mathcal{B}$ is congenial to $\mathcal{C}$ but $\mathcal{C}$ is not congenial to $\mathcal{B}$. On the other hand, we show that congeniality is transitive. Moreover, we consider the relation between two bases $\mathcal{B}$ and 
$\mathcal{C}$ being mutually congenial; that is, $\mathcal{B}$ is congenial to $C$ and $C$ is congenial to $\mathcal{B}$. We then show that mutually congenial bases have naturally isomorphic associated modules.

While mutual congeniality of two amenable bases $\mathcal{B}$ and $C$ suffices to conclude that $\mathcal{M}_{\mathcal{B}}$ and $\mathcal{M}_{C}$ are naturally isomorphic, one can also conclude that if $\mathcal{B}$ is properly congenial to $C$ ( $\mathcal{B}$ is congenial to $C$ but $C$ is not congenial to $\mathcal{B}$ ) then there exists a natural epimorphism from $\mathcal{M}_{\mathcal{B}}$ onto $\mathcal{M}_{\mathcal{C}}$. In the absence of mutual congeniality we cannot guarantee that the associated modules are isomorphic but to this moment we have not been able to find an argument to guarantee that they are not.

We explore various natural questions that arise in the context of proper congeniality. It seems that these questions will have different answers depending on the specific algebra in which they are considered. Our investigation was limited to the algebra of polynomials with coefficients in a field. Some consideration was given to semigroup and group algebras as well as to Leavitt Path Algebras but we have barely scratched the surface of what seem to be very deep problems.

\subsection{Preliminaries}

In this section we provide some basic modern and linear algebra definitions that are going to be used throughout this dissertation. These stated in standard references include [7], [9] and [15]. Then we extend the linear algebra ideas to countably infinite dimensional spaces as presented in [12] and [13].

In this dissertation rings are associative and have $1_{R}$ and modules are always unital.

Definition 1.2.1. By a ring we mean a nonempty set $R$ together with two binary operations (usually denoted as addition (+) and multiplication) such that

1. $(R,+)$ is an abelian group;

2. For all a, $b, c \in R,(a b) c=a(b c)$ (associative multiplication); 
3. For all $a, b, c \in R, a(b+c)=a b+a c$ and $(a+b) c=a c+b c$ (left and right distributive laws)

4. For all $a \in R, 1_{R} a=a 1_{R}$

Definition 1.2.2. Let $R$ be a ring. A (left) $R$-module is an additive abelian group $M$ together with a function $R \times M \rightarrow M$ ( the image if $(r, m)$ being denoted by $r m$ ) such that for all $r, s \in R$ and $m, n \in M$

1. $r(m+n)=r m+r n$.

2. $(r+s) m=r m+s m$

3. $r(s m)=(r s) m$

For 1, the identity in $R$,

4. $1_{R} m=m$ for all $m \in M$

If $R$ is a division ring, then a unitary $R$-module is called a (left) vector space.

Definition 1.2.3. Let $K$ be a commutative ring with identity. We say that $K$-algebra $A$ is a ring A such that

1. $(A,+)$ is a unitary K-module;

2. For all $k \in K$ and $a, b \in A k(a b)=(k a) b=a(k b)$

A $K$-algebra $A$ which is division ring is called a division $K$-algebra.

Next, we introduce the notion of direct sums and direct products, in the following definitions as they appear in [15].

Definition 1.2.4. Let $\left\{V_{1}, V_{2}, \cdots V_{n}\right\}$ be vector spaces over a field $K$. The direct sum of $\left\{V_{1}, \cdots V_{n}\right\}$, denoted by 


$$
V=V_{1} * V_{2} * \cdots * V_{n}
$$

is the vector space whose elements are ordered n-tuples:

$$
V=\left\{\left(v_{1}, \cdots, v_{n}: v_{i} \in V_{i}, i=1, \cdots, n\right\}\right.
$$

For all $k \in K$, with vector space operations (Addition is defined componentwise and multiplication by scalars is also defined componentwise) given by the formulas

$$
\left(u_{1}, \cdots, u_{n}\right)+\left(v_{1}, \cdots, v_{n}\right)=\left(u_{1}+v_{1}, \cdots, u_{n}+v_{n}\right)
$$

and

$$
k\left(v_{1}, \cdots, v_{n}\right)=\left(k v_{1}, \cdots, k v_{n}\right)
$$

In particular, The vector space $K^{n}$ is the direct sum of $n$ copies of $K$, that is

$$
K^{n}=K * \cdots * K
$$

where there are $n$ summands on the right-hand side.

We can generalize this construction to any family of vector spaces by the following: An ordered $n$-tuple $\left(v_{1}, \cdots, v_{n}\right)$ can be defined as a function $\phi:\{1, \cdots, n\} \rightarrow \bigcup V_{i}$ from the index set to the union of the spaces such that $\phi(i) \in V_{i}$.

Definition 1.2.5. Let $\mathcal{V}=\left\{V_{i}: i \in I\right\}$ be any family of vector spaces over $K$. The direct product of $\mathcal{V}$ is the vector space

$$
\prod_{i \in I} V_{i}=\left\{\phi: I \rightarrow \bigcup_{i \in I} V_{i}: \phi(i) \in V_{i}\right\}
$$

thought of as a subspace of the vector space of all functions from index I to $\bigcup V_{i}$.

Now we define a support of a function and it would be useful to restrict the set of functions to those with finite support.

Definition 1.2.6. Let $\mathcal{V}=\left\{V_{i}: i \in I\right\}$ be a family of vector spaces over $K$. The set

$$
\operatorname{supp}(\phi)=\{i \in I: \phi(i) \neq 0\}
$$

is the support of a function $\phi: I \rightarrow \bigcup V_{i}$.

Thus, a function $\phi$ has finite support if $\phi(i)=0$ for all but finitely many of $i \in I$. The direct sum of the family $\mathcal{V}$ is the vector space 


$$
\bigoplus_{i \in I} V_{i}=\left\{\phi: I \rightarrow \bigcup_{i \in I} V_{i}, \phi \text { has finite support }\right\}
$$

thought of as a subspace of the vector space of all functions from I to $\bigcup V_{i}$.

In particular, let $V_{i}=V$ for all $i \in I$ and let $V^{I}$ be denote the set of all functions from I to $V$ and $V^{(I)}$ denote the set of all functions in $V^{I}$ that have finite support, then

$$
\prod_{i \in I} V=V^{I} \text { and } \bigoplus_{i \in I} V=V^{(I)}
$$

Note that the direct product and the direct sum are the same for a finite family of vector spaces.

Definition 1.2.7. A vector space $V$ is a finite dimensional if it has a finite basis. All other vector spaces are infinite dimensional.

Definition 1.2.8. Let $V$ and $W$ be vector spaces (over $K$ ). We call a function $T: V \rightarrow W a$ linear transformation from $V$ to $W$ if, for all $v_{1}, v_{2} \in V$ and $k \in K$, we have

1. $T\left(v_{1}+v_{2}\right)=T\left(v_{1}\right)+T\left(v_{2}\right)$ and

2. $T\left(k v_{1}\right)=k T\left(v_{2}\right)$

If $T$ is surjective and injective we called $T$ an isomorphism linear transformation. For any vector space $V$ over $K$, we define the identity transformation $I_{V}: V \rightarrow V$ by $I_{V}(v)=v$ for all $v \in V$. It is clear that the identity transformation is an isomorphism.

Definition 1.2.9. A linear transformation $T: V \rightarrow W$ that is bijective (that is, injective and surjective) is called an isomorphism linear transformation.

Definition 1.2.10. Let $\mathcal{B}=\left\{u_{1}, u_{2}, \cdots, u_{n}\right\}$ be an ordered basis for a finite dimensional vector space $V$. For $v \in V$, let $k_{1}, k_{2}, \cdots, k_{n}$ be the unique scalars such that

$$
\sum_{i=1}^{n} k_{i} u_{i}
$$

we define the coordinate vector of $v$ relative to $\mathcal{B}$, denoted $[v]_{\mathcal{B}}$ by 


$$
[v]_{\mathcal{B}}=\left(\begin{array}{c}
k_{1} \\
k_{2} \\
\vdots \\
k_{n}
\end{array}\right)
$$

The correspondence $x \rightarrow[v]_{\mathcal{B}}$ provides us an isomorphism from $V$ to $K^{n}$.

Suppose $V$ and $W$ are finite-dimensional vector spaces with ordered bases $\mathcal{B}=\left\{v_{1}, v_{2}, \cdots, v_{n}\right\}$ and $C=\left\{w_{1}, w_{2}, \cdots, w_{m}\right\}$, respectively and let $T: V \rightarrow W$ be a linear transformation. Then for each $j, 1 \leq j \leq n$, there exist unique scalars $k_{i j} \in K, 1 \leq i \leq m$, such that

$$
T\left(v_{j}\right)=\sum_{i=1}^{m} k_{i j} w_{i} \text { for } 1 \leq j \leq n .
$$

Definition 1.2.11. Using the notation above, we call the $m \times n$ matrix $A$ defined by $A_{i j}=k_{i j}$ the matrix representation of $T$ relative to the ordered bases $(\mathcal{B}, C)$ and write $A=[T]_{\mathcal{B}}^{C}$. If $V=W$ and $\mathcal{B}=C$, then we simply write $[T]_{\mathcal{B}}$.

Notice that the $j$ th column of $A$ is simply $\left[T\left(v_{j}\right)\right]_{C}$. Matrix representations of linear transformations serve to yield representations of outputs via matrix multiplication from representations of inputs due to the identity. Thus we have the theorem

Theorem 1.2.1. Let $V$ and $W$ be finite dimensional vector space having ordered bases $\mathcal{B}$ and $\mathcal{C}$, respectively, and let $T: V \rightarrow W$ be linear. Them for each $v \in V$, we have $[T(v)]_{C}=[T]_{\mathcal{B}}^{C} \cdot[v]_{\mathcal{B}}$

Definition 1.2.12. Let $\mathcal{B}$ and $\mathcal{C}$ be two ordered bases for an finite-dimensional vector space $V$, the matrix $Q=\left[I_{V}\right]_{\mathcal{B}}^{C}$ is called a change of coordinate matrix. $Q$ changes $\mathcal{B}$-coordinates into C-coordinates. Namely, $[v]_{\mathcal{C}}=Q \cdot[v]_{\mathcal{B}}$

Definition 1.2.13. Let $V$ and $W$ be vector spaces, and let $T: V \rightarrow W$ be linear. If $\operatorname{Ker}(T)$ and $\operatorname{Im}(T)$ are finite-dimensional, then we define the nullity of $T$, denoted nullity $(T)$, and the rank of $T$, denoted $\operatorname{rank}(T)$, to be the dimensions of $\operatorname{Ker}(T)$ and $\operatorname{Im}(T)$, respectively. 
Theorem 1.2.2. Let $V$ and $W$ be vector spaces, and let $T: V \rightarrow W$ be linear. If $V$ is finite-dimensional, then

$$
\operatorname{nullity}(T)+\operatorname{rank}(T)=\operatorname{dim}(V)
$$

We conclude this section with an adaption of the previous linear algebra results to the countably infinite dimensional case as seen in [13] and [12].

\subsection{Matrix Representations of Linear Mappings on $K^{(\omega)}$}

We present the notion Matrix Representations of Linear Mappings on $K^{(\omega)}$ as it appears in [13]. The set of natural numbers $\mathbb{N}=\{0,1,2, \ldots\}$ (endowed its standard well ordering) is identified with the ordinal notation $\omega$. Let $K$ be a field and $K^{(\omega)}$ be the vector space of sequences in $K$ of only finitely many nonzero terms. The canonical basis of $K^{(\omega)}$ is denoted by $E=\left\{e_{i}\right\}_{i \in \omega}$, where $e_{i}=\left(\delta_{i j}\right)_{j \in \omega}$ and $\delta_{i j}$ is the Kronecker delta function. A row-finite $\omega \times \omega$ matrix $\mathrm{A}$ is a sequence of vectors in $K^{(\omega)}$ indexed by $\omega$ and denoted by $A=\left(A_{r}\right)_{r \in \omega}=\left(a_{r i}\right)_{(r, i) \in \omega \times \omega}$. Also, let $I=\left(e_{i}\right)_{i \in \omega}$ be the identity $\omega \times \omega$ matrix. A row $A_{r} \in K^{(\omega)}$ of $A$ is written as:

$$
A_{r}=\left(a_{r 0}, a_{r 1}, \ldots, a_{r \rho_{r}}, 0,0 \cdots\right)=\sum_{i=0}^{\rho_{r}} a_{r i} e_{i}
$$

The index $\rho_{r}$ is called rightmost index of $A_{r}$ (rightmost is the nonzero right leading entry of the row) and it is denoted by $l\left(A_{r}\right)$. We have $l\left(e_{i}\right)=i$ for $i \in \omega$, thus $l\left(e_{0}\right)=0$. Since $l(0)$ does not exist in $\omega$, the convention $l(0)=-1$ is adopted. The rightmost nonzero coefficient $a_{r \rho_{r}}$ of $A_{r}$ will be also called right leading coefficient of $A_{r}$.

We denote $\operatorname{Hom}_{K}\left(K^{(\omega)}\right)$ the space of linear mappings from $K^{(\omega)}$ to $K^{(\omega)}$ over $K$. Let $T \in \operatorname{Hom}_{K}\left(K^{(\omega)}\right)$. The null space and the range of $T$ is denoted by $\operatorname{Ker}(T)$ and $\operatorname{Im}(T)$ respectively. Let $\mathcal{B}=\left\{b_{i}\right\}_{i \in \omega}$ and $C=\left\{c_{j}\right\}_{j \in \omega}$, be bases of the domain and codomain of $T$ respectively. The matrix representation of $T$, relative to $(\mathcal{B}, \mathcal{C})$, is a row-finite matrix $A=\left(a_{i j}\right)_{(i, j) \in \omega \times \omega}$. The entries of $A$ are determined by $T\left(b_{i}\right)=\sum a_{i j} c_{j}$ and we shall referred to it as $A=[T]_{C}^{\mathcal{B}}$. 
On the other hand, if $A \in \mathbb{R F} \mathbb{M}_{\omega}(K)$, ( The set of all matrices where each row has only finitely many nonzero entries ) there is a unique linear extension of $b_{i} \rightarrow \sum a_{i j} c_{j}$ to $\operatorname{Hom}_{K}\left(K^{(\omega)}\right)$, call it $U$, such that $A=[U]_{\mathcal{C}}^{\mathcal{B}}$. A row $A_{i}=\left(a_{i j}\right)_{j \in \omega}$ of $A$ represents the element $U\left(b_{i}\right)$, relative to $C$. If $\mathcal{B}=C$ then the matrix representation of $U$, relative to $\mathcal{B}$ is denoted by $[U]_{\mathcal{B}}$.

The matrix multiplication of two matrices in $\mathbb{R} F \mathbb{M}_{\omega}(K)$ is defined in complete analogy with finite matrices, and results in a matrix in $\mathbb{R F M}_{\omega}(K)$. Moreover, If $\alpha \in K^{(\omega)}$, the mapping

$$
\begin{gathered}
T: K^{(\omega)} \rightarrow K^{(\omega)} \\
\alpha \rightarrow T(\alpha)=\alpha \cdot A
\end{gathered}
$$

Will be called the induced linear mapping by $A$. In this setting, $A$ is treated as a right operator and $A=[T]_{E}$, since

$$
T\left(e_{k}\right)=e_{k} \cdot A=\left(a_{k 0}, a_{k 1}, \ldots, a_{k \rho_{k}}, 0,0 \cdots\right)=\sum_{i=0}^{\rho_{k}} a_{k i} e_{i}
$$

Now we inroduce the notion of change of bases and left association as it appears in [13] $A$ is said to be non-singular or invertible If a left and a right inverse of $A \in \mathbb{R} \mathbb{F} \mathbb{M}_{\omega}(K)$ exist in $\mathbb{R F M}_{\omega}(K)$, then they necessarily coincide defining the two-sided inverse of $A$. Therefore, the existence of a left (resp. right) inverse implies that the induced linear mapping is surjective (respectively injective), whence the following Proposition:

Proposition 1.3.1. [13] Let $T \in \operatorname{Hom}_{K}\left(K^{(\omega)}\right)$. Then $T$ is an isomorphism if and only if $A=[T]_{E}^{E}$, is non-singular.

If $\mathcal{G} \subset K^{(\omega)}$ spans a subspace $\mathcal{Z}$ of $K^{(\omega)}$, we write $\operatorname{span}(\mathcal{G})=\mathcal{Z}$. The null space and the row space of $A \in \mathbb{R F M}_{\omega}(K)$ are defined by $N(A)=\operatorname{Ker}(T)=\left\{\alpha \in K^{(\omega)}: \alpha \cdot A=0\right\}$ and $R(A)=\operatorname{Im}(T)=\operatorname{span}\left(A_{r}\right)_{r \in \omega}$. The dimensions of these spaces are called nullity and rank of $A$, respectively, writing $\operatorname{nul}(A)=\operatorname{dim}(N(A)), \operatorname{rank}(A)=\operatorname{dim}(R(A))$. Let $\mathcal{D}=\left(d_{r}\right)_{r \in \omega}$ be basis of $K^{(\omega)}$, then $T \in \operatorname{Hom}_{K}\left(K^{(\omega)}\right)$ and $A=[T]_{\mathcal{B}}^{C}, B=[T]_{\mathcal{D}}^{C}$. Let also $\psi: \mathcal{B} \rightarrow \mathcal{D}$ be defined by $d_{r}=\psi\left(b_{r}\right)$ and $\psi\left(b_{r}\right)=\sum_{i} q_{r i} b_{i}$. As the unique linear extension of the bijection 
$\psi$, say $\varphi: K^{(\omega)} \rightarrow K^{(\omega)}$, is an isomorphism, the matrix $Q=\left(q_{r i}\right)_{(r, i) \in \omega \times \omega}=[\varphi]_{\mathcal{B}}^{\mathcal{B}}$ is nonsingular, called the matrix of passage from the basis $\mathcal{B}$ to the basis $\mathcal{D}$. The matrix $Q$ is just the matrix with respect to the basis $\mathcal{B}$ of the isomorphism $\varphi$. It also can be interpreted as the matrix of the identity mapping with respect to the basis $\mathcal{D}$ to the basis $\mathcal{B}$ (in that order). This passage of bases causes a change of the matrix representation of $T$ from $A$ to $B$, for a fixed basis $C$ of the codomain space, described by

$$
Q \cdot A=B \quad *
$$

Let $Q \in \mathbb{R F M}_{\omega}(K)$ be non-singular such that $*$ holds. Then $A, B$ is said to be left associates or row-equivalent and we write $A \sim B$. Formally $\sim$ is an equivalence relation on $\mathbb{R F M}_{\omega}(K)$, which generalizes the the well known notion of "row-equivalence" for finite matrices. A change of the domain basis gives rise to row equivalent matrix representations of a linear mapping and vise versa.

Definition 1.3.1. $A, B \in \mathbb{R} \mathbb{F} \mathbb{M}_{\omega}(K)$ are said to be:

1. Left associates if there exists a nonsingular matrix $Q \in \mathbb{R F} \mathbb{M}_{\omega}(K)$ such that $Q \cdot A=B$.

2. Row equivalent if $R(A)=R(B)$ and $\operatorname{nul}(A)=\operatorname{nul}(B)$. 


\section{Related Topics}

\subsection{Formal Calculus and Vertex Operator Algebra}

Formal calculus is an essential topic in vertex operator algebra theory. The notion of amenability in this dissertation and the notion of algebraic summablity in formal calculus seem to be similar at a first glance. In order to understand each topic, we shall provide a quick primer to vertex operator algebra and some basic elements of formal calculus. For more information, the reader should consult for [10].

Let $V$ be vector space. We denote by $V\left[\left[x, x^{-1}\right]\right]$ the vector space of (doubly infinite ) formal Laurent series in $x$ with coefficient in $V$. The vector spaces $W$ in vertex operator algebra are often in the form $W=$ End $V$, the algebra of endomorphisms of a vector space $V$. Now we state the definition of vertex operator algebra which is taken directly from [10].

Definition 2.1.1. A vertex algebra consists of a vector space $V$ equipped, first, with a linear map (the vertex operator map) $V \otimes V \rightarrow V\left[\left[x, x^{-1}\right]\right]$, or equivalently, a linear map

$$
\begin{aligned}
Y(\cdot, x): V & \rightarrow(\text { End } V)\left[\left[x, x^{-1}\right]\right] \\
v & \rightarrow Y(v, x)=\sum_{n \in \mathbb{Z}} v_{n} x^{-n-1}
\end{aligned}
$$

Notice that we are using the notation $v_{n}$ for the element of End $V$ which is the coefficient of $x^{-n-1}$ in the formal series $Y(v, x)$; (in particular, the notation $v_{n}$ does not refer to any sort of component of the vector $v$.) We call $Y(v, x)$ the vertex operator associated with $v$. We also have a distinguished element 1 of $\mathrm{V}$ (the vacuum vector). The following conditions are assumed for $u, v \in V$ : First, the truncation condition:

$$
u_{n} v=0 \text { for sufficiently large, }
$$

that is,

$$
Y(u, x) v \in V((x))
$$

Next, the following vacuum property: $Y(1, x)=1(1$ on the right being the identity operator); also, the creation property (whereby $Y(v, x)$ "creates" the vector v): 


$$
Y(v, x) 1 \in V[[x]] \text { and } \lim _{x \rightarrow 0} Y(v, x) 1=v
$$

(that is, $Y(v, x) 1$ involves only nonnegative integral powers of $x$ and the constant term is $v$ ); and finally, and most importantly, the Jacobi identity:

$$
x_{0}^{-1} \delta\left(\frac{x_{1}-x_{2}}{x_{0}}\right) Y\left(u, x_{1}\right) Y\left(v, x_{2}\right)-x_{0}^{-1} \delta\left(\frac{x_{2}-x_{1}}{-x_{0}}\right) Y\left(v, x_{2}\right) Y\left(u, x_{1}\right)=x_{2}^{-1} \delta\left(\frac{x_{1}-x_{0}}{x_{0} 2}\right) Y\left(Y\left(u, x_{0}\right) v, x_{2}\right) .(1)
$$

It is strait forward - and of course necessary- to check that when each expression in (1) is applied to any element if $\mathrm{V}$, the coefficient of each monomial in the three formal variables is finite sum of vectors. On the right-hand side, the notation $Y\left(\cdot, x_{2}\right)$ is understood to be extended in an obvious way to $V\left[\left[x_{0}, x_{0}^{-1}\right]\right]$; this makes clear the meaning of the "iterate" $Y\left(Y\left(u, x_{0}\right) v, x_{2}\right)$ of the two vertex operators $Y\left(v, x_{2}\right)$ and $Y\left(u, x_{0}\right)$.

In formal calculus, everything depends upon formal Laurent series of the type $\sum_{n \in \mathbb{Z}} a_{n} x^{n}$ and analogous formal series in several variables. The aim of both definitions is that for the product of elements to be well behaved. However, the summability is needed not only for formal product of finitely many formal series but also for formal sums of infinitely many formal series so they can be well defined. In our case the amenability is needed only to get a well-defined multiplication of finite series, which is an element from the algebra, times an infinite formal series, which is an element from the constructed module. Now, we define the summability property of formal calculus.

Definition 2.1.2. Let $V$ be a vector space and $\left(f_{i}\right)_{i \in I}$ be family in End $V$ (I an index set). we say that $\left(f_{i}\right)_{i \in I}$ is summable if for every $v \in V, f_{i} v=0$ for all but a finite number of $i \in I$. in this case we write the corresponding "sum" operator as

$$
\begin{gathered}
\sum_{i \in I} f_{i}: V \rightarrow V \\
v \rightarrow \sum_{i \in I} f_{i} v
\end{gathered}
$$

The idea of the notion of algebraic summability is to be used for the product of finite formal series and the sum of infinite formal series so they can be well defined. Therefore, a finite formal product and an infinite formal sum of formal series is defined if and only if 
the coefficient of every monomial in the formal product or sum in the formal expression is summable as we can see in the next definitions.

Definition 2.1.3. We say that $\sum_{i \in I} F_{i}(x)$ exists if for every $n \in \mathbb{Z}$, the family $\left(f_{i}\right)_{i \in I}$ is summable. We then set

$$
\sum F_{i}(x)=\sum_{n \in \mathbb{Z}}\left(\sum_{i \in I} f_{i}(n)\right) x^{n}
$$

Let $\left(F_{i}(x)\right)_{i=1}^{r}$ be finite family in End $V\left[\left[x, x^{-1}\right]\right]$, with

$$
F_{i}(x)=\sum n \in \mathbb{Z} f_{i}(n) x^{n}
$$

We say the product $F_{1}(x) \cdots F_{r}(x)$ exists if for every $n \in \mathbb{Z}$, the family

$$
\left(f_{1}\left(n_{1}\right) \cdots f_{r}\left(n_{r}\right)\right)_{n_{1}+\cdots+n_{r}=n}^{n_{1} \cdots n_{r}}
$$

is summable. We then set

$$
F_{1}(x) \cdots F_{r}(x)=\sum_{n \in \mathbb{Z}}\left(\sum_{n_{1}+\cdots+n_{r}=n} f_{1}\left(n_{1}\right) \cdots f_{r}\left(n_{r}\right)\right) x^{n} \in(\text { End } V)\left[\left[x, x^{-1}\right]\right]
$$

On the other hand, the idea of the notion of amenability is that the formal product of finite series times infinite series on a module that is produced by a basis will be understood to exist if and only if the coefficient of any monomial in the formal product is finite, that is that basis is amenable as we can see through this dissertation. For more details we refer the reader to [10].

\subsection{Semigroup Rings and Group Rings}

Here we present the notion of semigroup ring and group rings as it appears in [11]. Let $G$ be a multiplicative group (finite or infinite) and $R$ be a ring. We construct an R-module, with elements of $\mathrm{G}$ as a basis, and then use the operations in both $G$ and $R$ to define a ring structure on it. To do so, let RG to be the set of all formal linear combinations of the form

$$
\alpha=\sum_{g \in G} a_{g} g
$$

with $\alpha_{g} \in R$ and $\alpha_{g}=0$ for almost everywhere, that is, $\alpha$ has only finitely many of the coefficients of $a_{g}$ is nonzero. whenever convenient, we may also write $\alpha$ in the form

$$
\alpha=\sum_{g \in G} a_{g} g
$$


Given an element $\alpha=\sum_{g \in G} a_{g} g$ we define the support of $\alpha$ to be the subset of elements in $G$ that they really accrue in the representation of $\alpha$, that is:

$$
\operatorname{supp}(\alpha)=\left\{g \in G: a_{g} \neq 0\right\}
$$

Notice that it follows from our definition that given two elements, $\alpha=\sum_{g} \in G a_{g} g$ and $\beta=\sum_{g \in G} b_{g} g \in R G$, we have that $\alpha=\beta$ if and only if $a_{g}=b_{g}, \forall g \in G$.

We define the addition of two elements in $R G$ componentwise:

$$
\left(\sum_{g \in G} a_{g} g\right)+\left(\sum_{g \in G} b_{g} g\right)=\sum_{g} \in G\left(a_{g}+b_{g}\right) g
$$

Also, given two elements $\alpha=\sum_{g \in G} a_{g} g$ and $\beta=\sum_{g \in G} b_{g} g$ in $R G$ we define the multiplication by

$$
\alpha \cdot \beta=\sum_{g, h \in G} a_{g} b_{h} g h
$$

Reordering the terms in the formula above we can write the product by

$$
\alpha \cdot \beta=\sum_{c \in G} c_{u} u
$$

where

$$
c_{u}=\sum_{g h=u} a_{g} b_{h}
$$

It is easy to verify that that, with the operations above, $R G$ is a ring, which has a unity; namely, the element $1=\sum_{g \in G} u_{g} g$ where the coefficient corresponding to the unite element of the group is equal to 1 and $u_{g}=0$ for every other element $g \in G$

We can also define a product of elements in $R G$ by elements $\lambda \in R$ as

$$
\lambda\left(\sum_{g \in G} a_{g} g\right)=\sum_{g \in G} \lambda\left(a_{g} g\right)
$$

Again it is easy to verify that $R G$ is an $R$-module. Actually, if $R$ is commutative, it follows that $R G$ is an algebra over $R$.

We can define an embedding $i: G \rightarrow R G$ by assigning to each element $h \in G$ the element $i(x)=\sum_{g \in G} a_{g} g$, where $a_{x}=1$ and $a_{g}=0$ if $g \neq h$, we may thus regard $G$ as a subset of $R G$. With this identification in mind, we can say that $G$ is a basis of $R G$ over $R$. As in immediate consequence we see that, if $R$ is commutative, them the rank of agree 
module over $R$ is well-define. Thus if $G$ is finite, we can state that the $\operatorname{rank}(R G)$ over $R$ is precisely $|G|$.

\subsection{Hamel Bases}

In this section we explain the notion of Hamel Basis and compare it with other bases. We begin with the definition of Hamel bases, which are the bases that we are familiar with in linear algebra as it appears in [8].

Definition 2.3.1. (Hamel Basis). Let $V$ be a vector space. A sequence of vectors $\left\{x_{i}\right\}_{i \in I}$ is a Hamel basis for $V$ if

- the finite linear span of $\left\{x_{i}\right\}_{i \in I}$ is $V$, i.e., $\operatorname{span}\left\{x_{i}\right\}=V$, and

- $\left\{x_{i}\right\}$ is finitely linear independent.

Note that the index set $I$ of Hamel basis need not to be countable. Equivalent formulations of Definition 2.3.1 are that $\left\{x_{i}\right\}_{i \in I}$ is a Hamel basis for $V$ if and only if every nonzero vector $x \in V$ can be written as $x=\sum_{k=1}^{N} c_{k} x_{i_{k}}$ for some unique choice indices $i_{1}, \cdots, i_{N} \in I$ and unique nonzero scalars $c_{1}, \cdots, c_{N}$, or that every $x \in V$ can be written uniquely as $x=\sum_{i \in I} a_{i}(x) x_{i}$ for some unique choice of scalars $a_{i}(x)$

In finite-dimensional linear algebra, a Hamel basis is usually just called a "basis". Thus, the definition of a Hamel basis, as given in Definition 2.3.1, is made entirely in terms of finite linear combinations of vectors. Every vector space has a Hamel basis. The proof uses the Axiom of Choice in the form of Zorn's Lemma. In addition, all Hamel bases for a given vector space have the same cardinality, which we call the Hamel dimension of the space. It is highly convenient to use Hamel bases for finite-dimensional vector spaces and vector spaces with countably infinite dimension. For example, the set $\mathcal{B}=\left\{x^{i}: i \geq 0\right\}$ is a Hamel basis for the vector space of polynomials. 
However, For infinite dimensional vector spaces, we only know that their Hamel bases exist because of the Axiom of Choice. Even if we know that every vector space has a Hamel basis, these bases are difficult to describe since there is no practical way in general to construct such bases. The obvious example is $\mathbb{R}$ as a vector space over $\mathbb{Q}$ where the Hemal basis of the vector space $\mathbb{R}$ over $\mathbb{Q}$ is uncountable.

Similarly, from the perspective of real analysis, a Hamel basis for normed spaces, for example infinite-dimensional Banach space, is necessarily uncountable "Baire Category Theorem." Hence, new notions of infinite bases were introduced that are more appropriate for infinite-dimensional normed spaces. This new notion of bases allow the use of "infinite linear combinations" instead of using finite linear combinations to which Hamel bases are restricted, provided that the infinite sum of vectors are compatible. Thus, seeing that the notion of convergence in normed spaces is applicable, infinite series can be constructed in order to make sense of an infinite sum of vectors in different types of normed spaces. This leads to the notion of Schauder Bases. 


\section{The Central Notion}

\subsection{Introduction}

Let $K$ be a field and $R$ be an infinite dimensional $K$-algebra with basis $\mathcal{B}$. We consider the feasibility to construct an $R$-module $\mathcal{M}$ from the basis $\mathcal{B}$. Let $\mathcal{M}=\{\phi: \mathcal{B} \rightarrow K\}$ the set of all functions from $\mathcal{B}$ to $\mathcal{M}$. Then $\mathcal{M}$ is a $K$-vector space where, for all $b \in \mathcal{B}$ and $k \in K$, we define $\phi+\psi \rightarrow \phi(b)+\psi(b)$ and $k \phi: b \rightarrow k[\phi(b)]$. We may write

$$
\phi=(\phi(b))_{b \in \mathcal{B}}=\sum_{b \in \mathcal{B}} \phi(b) b
$$

whenever convenient, we may also write $\phi$ in the form

$$
\phi=\sum_{b \in \mathcal{B}} \phi_{b} b .
$$

Remark. We would like to define a product $b \in \mathcal{B}$ of elements of $R$ with elements from $\mathcal{M}$. For $b, c \in \mathcal{B}$ and $k \in K$, define

$$
[(k b) \phi](c)=\sum_{d \epsilon_{b} \mathcal{B}_{c}} k(b d)_{c} \phi(d)
$$

where ${ }_{b} \mathcal{B}_{c}=\left\{d \in \mathcal{B}:(b d)_{c} \neq 0\right\}$.

We construct modules with respect to some bases under some conditions. We start with the basic basis, which is closed under product, and the cancelation property is valid. A more general basis could have one more condition; the product of two elements of that basis might be zero. We defined the most general basis we considered as the "amenable basis" as in Lemma 1.4.1.

We begin with the following useful Definitions.

Definition 3.1.1. Let $\mathcal{B}$ be a basis of $R$ we say that $\mathcal{B}$ is amenable if for every $b, c \in \mathcal{B}$ there exist only finitely many $d \in \mathcal{B}$ such that $(b d)_{c} \neq 0$, In other words the set ${ }_{b} \mathcal{B}_{c}=\left\{d \in \mathcal{B}:(b d)_{c} \neq 0\right\}$ is finite.

Let $\mathcal{B}$ and $C$ be bases of $R$ we say that $\mathcal{B}$ is amenable to $C$ if for every $b \in \mathcal{B}$ and $c \in C$ there exist only finitely many $d \in C$ such that $(b d)_{c} \neq 0$, In other words the set ${ }_{b} \mathcal{C}_{c}=\left\{d \in C:(b d)_{c} \neq 0\right\}$ is finite. 
Let $\mathcal{B}$ be amenable basis of $R$ and for $r \in R$ let $L_{r}$ be a linear transformation from $R$ to $R$ defined by $L_{r}=r b$ for all $b \in \mathcal{B}$. Then for all $r \in R$ the matrix $\left[L_{r}\right]_{\mathcal{B}}$ is row-finite.

Definition 3.1.2. For a field $K$ and basis $\mathcal{B}, K^{(\mathcal{B})}$ will denote the direct sum of copies of $K$ and the symbol $K^{\mathcal{B}}$ the direct product of $K$.

\subsection{Modules Constructed from Amenable Bases}

The condition that $\mathcal{B}$ is amenable guarantees that we can make the set $K^{\mathcal{B}}$ into a module (Which we call $\mathcal{M}$ ) as in Lemma.1. For instant, If $\mathcal{B}$ is closed under products i.e. For every $b_{1}$ and $b_{2}$ in $\mathcal{B}$ then $b_{1} b_{2} \in \mathcal{B}$, and for any $b_{1}, b_{2}$ and $b_{3}$ in $\mathcal{B}$, if $b_{1} b_{2}=b_{1} b_{3} \Rightarrow b_{2}=b_{3}$ ,then ${ }_{R} \mathcal{M}$ is a module. In Particular, the bases in group algebras are closed under products. More generally, for every $b_{1}$ and $b_{2}$ in $\mathcal{B}$ either $b_{1} b_{2} \in \mathcal{B}$ or $b_{1} b_{2}=0$ and for any $b_{1}, b_{2}$ and $b_{3}$ in $\mathcal{B}$, if $b_{1} b_{2}=b_{1} b_{3} \Rightarrow b_{2}=b_{3}$, then ${ }_{R} \mathcal{M}$ is a module. In Particular, the bases in Leavitt Path Algebras as we will explain in more details in section.3.

Lemma 3.2.1. Let $\mathcal{B}$ be a basis of $R$ and if $\mathcal{B}$ is amenable then ${ }_{R} \mathcal{M}$ is a module.

Proof. We want to show that ${ }_{R} \mathcal{M}$ is a module. So we show that the product $r m(r \in R, m \in M)$ is well-defined.

$\left[\left(\sum_{i=1}^{n} k_{i} b_{i}\right) \phi\right](c)=\sum_{i=1}^{n}\left(\sum_{d \epsilon_{b} \mathcal{B}_{c}} k_{i}\left(b_{i} d\right)_{c} \phi(d)\right)$ this is a well defined.

- $\left[\sum_{i=1}^{n} k_{i} b_{i} \cdot(\phi+\psi)\right](c)=\sum_{i=1}^{n}\left(\sum_{d \epsilon_{i} \mathcal{B}_{c}} k_{i}\left(b_{i} d\right)_{c}(\phi+\psi)(d)\right)$

$=\sum_{i=1}^{n}\left(\sum_{d \epsilon_{i} \mathcal{B}_{c}} k_{i}\left(b_{i} d\right)_{c} \phi(d)\right)+\sum_{i=1}^{n}\left(\sum_{d \epsilon_{i} \mathcal{B}_{c}} k_{i}\left(b_{i} d\right)_{c} \psi(d)\right)$

$=\left[\left(\sum_{i=1}^{n} k_{i} b_{i}\right) \phi\right](c)+\left[\left(\sum_{i=1}^{n} k_{i} b_{i}\right) \psi\right](c)$

- $\left[\left(\sum_{i=1}^{n} k_{i} b_{i}+\sum_{j=1}^{m} k_{j}^{\prime} b_{j}\right) \phi\right](c)=\left[\left(\sum_{i=1}^{n} k_{i} b_{i}\right) \phi\right](c)+\left[\left(\sum_{j=1}^{m} k_{j}^{\prime} b_{j}\right) \phi\right](c)$

$=\sum_{i=1}^{n}\left(\sum_{d \epsilon_{b_{i}} \mathcal{B}_{c}} k_{i}\left(b_{i} d\right)_{c} \phi(d)\right)+\sum_{j=1}^{m}\left(\sum_{d \epsilon_{j} \mathcal{B}_{c}} k_{j}^{\prime}\left(b_{j} d\right)_{c} \phi(d)\right)$

$=\left[\sum_{i=1}^{n} k_{i} b_{i} \phi\right](c)+\left[\sum_{j=1}^{m} k_{j} b_{j} \phi\right](c)$ 
- $\left[\left(\sum_{i=1}^{n} k_{i} b_{i} \cdot \sum_{j=1}^{m} k_{j}^{\prime} b_{j}\right) \phi\right](c)=\left[\sum_{i=1}^{n} k_{i} b_{i}\left(\left(\sum_{j=1}^{m} k_{j}^{\prime} b_{j}\right) \phi\right)\right](c)$

Now in the theorem below we show that the following statements are equivalent: A basis $\mathcal{B}$ is amenable to $C$, all bases are amenable to $C$ and $C$ is amenable to itself. As a result, we will simply say that $C$ is amenable if one of the previous statements is satisfied.

Theorem 3.2.2. Let $C$ and $\mathcal{B}$ be bases. Then the Following are equivalent:

i) There exists a basis $\mathcal{B}$ such that $\mathcal{B}$ is amenable to $C$.

ii) For every basis $\mathcal{B}, \mathcal{B}$ is amenable to $\mathcal{C}$.

iii) $C$ is amenable to itself.

Proof. ii) $\Rightarrow$ i) clear

iii) $\Rightarrow$ ii) Suppose that $C$ is amenable to itself, means that for fixed $c_{1}, c_{2} \in C$ the set ${ }_{c_{1}} C_{c_{2}}=\left\{v \in C:\left(c_{1} v\right)_{c_{2}} \neq 0\right\}$ is finite. We claim that for fixed $b \in \mathcal{B}$ and $c \in C$ the set ${ }_{b} C_{c}=\left\{v \in C:(b v)_{c} \neq 0\right\}$ is finite. Since $C$ is a basis, we can write $b$ as a linear combination of $C$ as $b=\sum_{i=1}^{n}(b)_{c_{i}} c_{i}$ where $c_{i} \in C, 1 \leq i \leq n$.

Since $C$ is amenable to itself then for $1 \leq i \leq n$ the sets ${ }_{c_{i}} C_{c}=\left\{v_{c_{i}} \in C:\left(c_{i} v_{c_{i}}\right)_{c} \neq 0\right\}$ are finite. Let $\mathfrak{U}=\cup_{i=1 c_{i}}^{n} \mathcal{C}_{c}$. We want to show that ${ }_{b} \mathcal{C}_{c} \subseteq \mathfrak{U}$.

Now suppose $v \notin \mathfrak{U}$ such that $(b v)_{c} \neq 0$.

$$
\begin{aligned}
& \Rightarrow b v=\sum_{i=1}^{n}(b)_{c_{i}}\left(c_{i} v\right) \\
& \Rightarrow(b v)_{c}=\sum_{i=1}^{n}(b)_{c_{i}}\left(c_{i} v\right)_{c}
\end{aligned}
$$

Since $(b v)_{c} \neq 0$, there exist $c_{i}$ such that $\left(c_{i} v\right)_{c} \neq 0$ which is a contradiction. Therefore if $v \in{ }_{b} C_{c}$ then $v \in \mathfrak{U}$, but $\mathfrak{U}$ is finite then the set ${ }_{b} C_{c}$ is finite as claimed.

i) $\Rightarrow$ iii) similar to $i i i) \Rightarrow i i) \square$

Not all bases of an algebra are amenable as we can see in the following examples.

Example 3.2.1. Consider the polynomial ring $\mathbb{F}[x]$ over a field $\mathbb{F}$ and let $C=\{1\} \cup\left\{x^{i}+\right.$ $\left.x^{i+1}: i \geq 0\right\}$ and let $\mathcal{D}=\left\{\sum_{i=0}^{n} x^{i}: n \geq 0\right\}$. It is easy to see that $\mathcal{C}$ and $\mathcal{D}$ are bases of 
$\mathbb{F}[x]$ and $\mathcal{C}$ is amenable, however, $\mathcal{D}$ is not amenable. To show that $\mathcal{D}$ is not amenable, fix $d_{1}=1+x$ and $d_{2}=1 \in \mathcal{D}$, then the set $\left\{v \in \mathcal{D}:((1+x) v)_{1_{\mathcal{D}}} \neq 0\right\}$ is infinite, since $(1+x)\left(\sum_{i=0}^{n} x^{i}\right)=(1) \sum_{i=0}^{n+1} x^{i}+(1) \sum_{i=0}^{n} x^{i}+(-1) 1_{\mathcal{D}}$.

Take $x \in R$ and let $T_{x}$ be the linear transformation from $\mathbb{F}^{(\mathcal{C})}$ to $\mathbb{F}^{(\mathcal{C})}$ defined by left multiplication by $x$. Clearly the representation $\left[T_{x}\right]_{C}$ is row finite. Since $T_{x}(1)=(x+1)-1$ and $T_{x}\left(x^{i}+x^{i+1}\right)=x^{i+1}+x^{i+2}$ then we have

$$
\left[T_{x}\right]_{C}=\left(\begin{array}{ccccccc}
-1 & 0 & 0 & 0 & \cdots & 0 & \cdots \\
1 & 0 & 0 & 0 & \cdots & 0 & \cdots \\
0 & 1 & 0 & 0 & \cdots & 0 & \cdots \\
0 & 0 & 1 & 0 & \cdots & 0 & \cdots \\
0 & 0 & 0 & 1 & \cdots & 0 & \cdots \\
\vdots & \vdots & \vdots & \vdots & \vdots & \vdots &
\end{array}\right)
$$

On the other hand, take $(x+1) \in R$ and let $T_{(x+1)}$ be the linear transformation from $\mathbb{F}^{(\mathcal{D})}$ to $\mathbb{F}^{(\mathcal{D})}$ defined by left multiplication by $(x+1)$. Then the matrix representation $\left[T_{(x+1)}\right]_{\mathcal{D}}$ is not row finite. Since

$$
\begin{gathered}
T_{(x+1)}(1)=(x+1) \text { and } T_{(x+1)}\left(\sum_{i=0}^{n} x^{i}\right)=(1) \sum_{i=0}^{n+1} x^{i}+(1) \sum_{i=0}^{n} x^{i}+(-1) 1_{\mathcal{D}} \text { Then we have } \\
{\left[T_{(x+1)}\right]_{\mathcal{D}}=\left(\begin{array}{ccccccc}
-1 & -1 & -1 & -1 & \cdots & -1 & \cdots \\
1 & 1 & 0 & 0 & \cdots & 0 & \cdots \\
0 & 1 & 1 & 0 & \cdots & 0 & \cdots \\
0 & 0 & 1 & 1 & \cdots & 0 & \cdots \\
0 & 0 & 0 & 1 & \cdots & 0 & \cdots \\
\vdots & \vdots & \vdots & \vdots & \vdots & \vdots &
\end{array}\right)}
\end{gathered}
$$

Example 3.2.2. Consider the Laurent polynomial ring $\mathbb{F}\left[x, x^{-1}\right]$ over a field $\mathbb{F}$ and let $C=\{1\} \cup\left\{x^{i}+x^{i+1}: i \in \mathbb{Z}\right\}, \mathcal{D}=\{1\} \cup\left\{x^{i}+(-1)^{j} x^{-i}, i \in \mathbb{Z} /\{0\}, j \in\{0,1\}\right\}$ and $\mathcal{E}=\{1\} \cup\left\{1+x^{i}, i \in \mathbb{Z} /\{0\}\right\}$. It is easy to see that $\mathcal{C}, \mathcal{D}$ and $\mathcal{E}$ are bases of $\mathbb{F}\left[x, x^{-1}\right]$. In addition, $\mathcal{C}$ and $\mathcal{D}$ are amenable bases, however, $\mathcal{E}$ is not amenable. To show that $\mathcal{E}$ is 
not amenable, take $x \in \mathbb{F}\left[x, x^{-1}\right]$ and $e=1 \in \mathcal{E}$, then the set $\left\{v \in \mathcal{E}:(x v)_{1_{\mathcal{E}}} \neq 0\right\}$ is infinite, since $x \cdot(1)=(x+1)-1$ and $x \cdot\left(1+x^{i}\right)=(x+1)+\left(x^{i+1}+1\right)+(-2) 1_{\mathcal{E}}$ for every $i \in \mathbb{Z}^{+}$.

Take $x \in R$ and let $T_{x}$ be the linear transformation from $\mathbb{F}^{(\mathcal{E})}$ to $\mathbb{F}^{(\mathcal{E})}$ defined by left multiplication by $x$. Then the representation matrix $\left[T_{x}\right]_{\mathcal{E}}$ is not row finite. Since

$$
T_{x}(1)=(x+1)-1 \text { and } T_{x}\left(1+x^{i}\right)=(x+1)+\left(x^{i+1}+1\right)+(-2) 1_{\mathcal{E}} \text { for every } i \in \mathbb{Z}^{+} . \text {then }
$$

we have

$$
\left[T_{x}\right]_{\mathcal{E}}=\left(\begin{array}{cccccccccccc}
\mathbf{- 1} & 0 & \mathbf{- 2} & \mathbf{- 2} & \mathbf{- 2} & \mathbf{- 2} & \mathbf{- 2} & \mathbf{- 2} & \mathbf{- 2} & \cdots & \mathbf{- 2} & \cdots \\
\mathbf{1} & \mathbf{1} & \mathbf{1} & \mathbf{1} & \mathbf{1} & \mathbf{1} & \mathbf{1} & \mathbf{1} & \mathbf{1} & \cdots & \mathbf{1} & \cdots \\
0 & 0 & 0 & 0 & \mathbf{1} & 0 & 0 & 0 & 0 & \cdots & 0 & \cdots \\
0 & \mathbf{1} & 0 & 0 & 0 & 0 & 0 & 0 & 0 & \cdots & 0 & \cdots \\
0 & 0 & 0 & 0 & 0 & 0 & \mathbf{1} & 0 & 0 & \cdots & 0 & \cdots \\
0 & 0 & 0 & \mathbf{1} & 0 & 0 & 0 & 0 & 0 & \cdots & 0 & \cdots \\
0 & 0 & 0 & 0 & 0 & 0 & 0 & 0 & \mathbf{1} & \cdots & 0 & \cdots \\
0 & 0 & 0 & 0 & 0 & \mathbf{1} & 0 & 0 & 0 & \cdots & 0 & \cdots \\
\vdots & \vdots & \vdots & \vdots & \vdots & \vdots & \vdots & \vdots & \vdots & \vdots & \vdots & \vdots
\end{array}\right)
$$




\section{IsOMORPhic Modules}

\subsection{Introduction}

Our goal of this dissertation is to investigate how different amenable bases relate to one another and wether the various amenable bases yield isomorphic module structures. Let $R$ be an infinite dimensional $K$-algebra and let $\mathcal{B}$ and $C$ be two bases of $R$. From these two bases we can produce two $R$-Modules $\mathcal{M}_{\mathcal{B}}, \mathcal{M}_{C}$ with respect $\mathcal{B}, \mathcal{C}$ respectively. Define next what we mean by that these two modules are naturally isomorphic.

Definition 4.1.1. Let $R$ be an infinite dimensional $K$-algebra and let $\mathcal{B}$ and $C$ be two bases of $R$. From these two bases we can produce two R-Modules $\mathcal{M}_{\mathcal{B}}, \mathcal{M}_{C}$ depends on $\mathcal{B}, C$ respectively. A homomorphism $\phi: M_{B} \rightarrow M_{C}$, said to be $\phi$ is natural if and only if $\left.\phi\right|_{A}=1_{A}$. If $\phi$ is an isomorphism then it is a natural isomorphism.

These conditions on the bases leads to the definition of a Congeniality relation between bases $\mathcal{B}$ and $C$.

Definition 4.1.2. We say that a basis $\mathcal{B}$ is congenial to a basis $C$ if every element $c \in C$ there exits only finitely many elements $b \in \mathcal{B}$, when represented in terms of $C$ use a non-zero coefficient for $c$. We mean that $\forall c \in C \Rightarrow\left|\mathcal{B}_{c}\right|=\left|\left\{b \in \mathcal{B}:(b)_{c} \neq 0\right\}\right|<\infty$. We say that $\mathcal{B}$ and $C$ are mutually congenial if $\mathcal{B}$ is congenial to $\mathcal{C}$ and $\mathcal{C}$ is congenial to $\mathcal{B}$.

Let $\mathcal{B}:=\left(b_{i}\right)_{i \in I}$ and $C:=\left(c_{i^{\prime}}\right)_{i^{\prime} \in I}$ be two bases of $R$, and $B$ is congenial to $C$ we may write $b_{i}=\sum \theta_{i i^{\prime}} c_{i}^{\prime}$. Then the matrix $\left[I_{R}\right]_{\mathcal{B}}^{C}=\left(\theta_{i i^{\prime}}\right)_{\left(i, i^{\prime}\right)}$ is a row-column finite matrix. Also $\left[I_{R}\right]_{\mathcal{B}}^{C}$ is invertible with inverse $\left(\left[I_{R}\right]_{\mathcal{B}}^{C}\right)^{-1}=\left[I_{R}\right]_{C}^{\mathcal{B}}$ column finite. Hence $\left(\left[I_{R}\right]_{\mathcal{B}}^{C}\right)^{-1}$ may be not row-finite as in Example 4.1.1

Lemma 4.1.1. $B$ is congenial to $C$ and $C$ is congenial to $B$ if and only if $\left[I_{R}\right]_{B}^{C}$ is an invertible row-column finite with inverse $\left[I_{R}\right]_{C}^{B}$ row-column finite. 
Example 4.1.1. Let $\mathcal{B}=\left\{x^{i}: i \geq 0\right\}$ and $C=\{1\} \cup\left\{x^{i}+(-1)^{j} x^{i}: i \geq 0, j \in\{0,1\}\right\}$. We can see that $\mathcal{B}$ is congenial to $C$ then we have

$\left[I_{R}\right]_{C}^{\mathcal{B}}$ is row-column finite.

$$
\left[I_{R}\right]_{C}^{\mathcal{B}}=\left(\begin{array}{ccccccccc}
1 & 1 & 1 & 0 & 0 & 0 & 0 & 0 & \cdots \\
0 & 1 & -1 & 0 & 0 & 0 & 0 & 0 & \cdots \\
0 & 0 & 0 & 1 & 1 & 0 & 0 & 0 & \cdots \\
0 & 0 & 0 & 1 & -1 & 0 & 0 & 0 & \cdots \\
0 & 0 & 0 & 0 & 0 & 1 & 1 & 0 & \cdots \\
0 & 0 & 0 & 0 & 0 & 1 & -1 & 0 & \cdots \\
\vdots & \vdots & \vdots & \vdots & \vdots & \vdots & \vdots & \vdots &
\end{array}\right)
$$

In addition, we see that $\mathcal{C}$ is congenial to $\mathcal{B}$ then we have

$\left[I_{R}\right]_{\mathcal{B}}^{C}$ is row-column finite.

$$
\left[I_{R}\right]_{C}^{\mathcal{B}}=\left(\begin{array}{ccccccccc}
1 & 0 & 0 & 0 & 0 & 0 & 0 & 0 & \cdots \\
1 & 1 & 0 & 0 & 0 & 0 & 0 & 0 & \cdots \\
1 & -1 & 0 & 0 & 0 & 0 & 0 & 0 & \cdots \\
0 & 0 & 1 & 1 & 0 & 0 & 0 & 0 & \cdots \\
0 & 0 & 1 & -1 & 0 & 0 & 0 & 0 & \cdots \\
0 & 0 & 0 & 0 & 1 & 1 & 0 & 0 & \cdots \\
0 & 0 & 0 & 0 & 1 & -1 & 0 & 0 & \cdots \\
\vdots & \vdots & \vdots & \vdots & \vdots & \vdots & \vdots & \vdots &
\end{array}\right)
$$

Example 4.1.2. Let $\mathcal{B}=\left\{x^{i}: i \geq 0\right\}$ and $\mathcal{C}=\{1\} \cup\left\{x^{i}+x^{i+1}: i \geq 0\right\}$. $\mathcal{B}$ is congenial to $C$ then we have

$$
\left[I_{R}\right]_{C}^{\mathcal{B}}=\left(\begin{array}{cccccccc}
1 & 1 & 0 & 0 & 0 & \cdots & 0 & \cdots \\
0 & 1 & 1 & 0 & 0 & \cdots & 0 & \cdots \\
0 & 0 & 1 & 1 & 0 & \cdots & 0 & \cdots \\
0 & 0 & 0 & 1 & 1 & \cdots & 0 & \cdots \\
\vdots & \vdots & \vdots & \vdots & \vdots & \vdots & \vdots &
\end{array}\right)
$$


$\left[I_{R}\right]_{C}^{\mathcal{B}}$ is row-column finite.

However, $C$ is not congenial to $\mathcal{B}$ then we have

$\left[I_{R}\right]_{\mathcal{B}}^{C}$ is column finite but not row finite.

$$
\left[I_{R}\right]_{\mathcal{B}}^{C}=\left(\begin{array}{cccccc}
1 & -1 & 1 & 1 & -1 & \cdots \\
0 & 1 & -1 & 1 & -1 & \cdots \\
0 & 0 & 1 & -1 & 1 & \cdots \\
0 & 0 & 0 & 1 & -1 & \cdots \\
\vdots & \vdots & \vdots & \vdots & \vdots &
\end{array}\right)
$$

Notice: Congeniality relation is not symmetric. For example, Let $\mathcal{B}=\left\{x^{i}: i \geq 0\right\}$ and $C=\{1\} \cup\left\{x^{i}+x^{i+1}: i \geq 0\right\} . \mathcal{B}$ is congenial to $C$ but $C$ is not congenial to $\mathcal{B}$. However, the transitivity property is satisfied as we prove in the Theorem below. Before that, we prove useful Lemma.

Alternatively, mutually congeniality is an equivalent relation. Since it is reflexive, symmetric and transitive.

Lemma 4.1.2. Let $\mathcal{B}, C$ and $\mathcal{D}$ be bases of $R . \forall d \in \mathcal{D}$ we have that $\mathcal{B}_{d} \subseteq \bigcup_{c \in C_{d}} \mathcal{B}_{c}$.

Proof. Take $b \in \mathcal{B}_{d}$ and suppose $b \notin \bigcup_{c \in C_{d}} \mathcal{B}_{c} \Rightarrow b \in \bigcap_{c \in C_{d}} \mathcal{B}_{c}^{c}=\left\{x \in \mathcal{B}:(x)_{c}=\right.$ $\left.0, \forall c \in \mathcal{C}_{d}\right\}$. Since $\mathcal{D}$ is a basis, we write $b$ as a linear combination of $\mathcal{D}$ as $b=\sum_{z \in \mathcal{D}}(b)_{z} z$. Let $Z=\operatorname{supp}(b)=\left\{z_{1}, \cdots, z_{t-1}, z_{t}\right\}$, where $z_{t}=d$, we can see that $(b)_{d} \neq 0$. Also we write $z_{i}, 1 \leq i \leq t$, as a linear combinations of $C$ as $z_{i}=\sum_{y \in C}\left(z_{i}\right)_{y} y$.

$$
\begin{aligned}
& b=\sum_{i=1}^{t}(b)_{z_{i}} z_{i} \\
& =\sum_{i=1}^{t}(b)_{z_{i}}\left[\sum_{y \in C}\left(z_{i}\right)_{y} y\right] \\
& =\sum_{i=1}^{t}\left[\sum_{y \in C}(b)_{z_{i}}\left(z_{i}\right)_{y} y\right] \\
& =\sum_{y \in C}\left[\sum_{i=1}^{t}(b)_{z_{i}}\left(z_{i}\right)_{y}\right] y,
\end{aligned}
$$

Thus $(b)_{y}=\sum_{i=1}^{t}(b)_{z_{i}}\left(z_{i}\right)_{y}$

So if $y \in C_{d}$ then $\sum_{i=1}^{t}(b)_{z_{i}}\left(z_{i}\right)_{y}=(b)_{y}=0$, since $b \notin B_{c}$ for all $c \in C_{d}$. Thus $y \notin C_{d}$ then we have $(y)_{d}=0$.Write $y$ as linear combination of $\mathcal{D}, y=\sum_{z \in \mathcal{D}}(y)_{z} z$. 
$b=\sum_{y \notin C_{d}}(b)_{y} y=\sum_{y \notin C_{d}}(b)_{y} \sum_{z \in \mathcal{D}}(y)_{z} z=\sum_{z \in \mathcal{D}}\left[\sum_{y \notin C_{d}}(b)_{y}(y)_{z}\right] z$

Therefore we have $0 \neq(b)_{d}=\sum_{y \notin C_{d}}(b)_{y}(y)_{d}$ but $(y)_{d}=0$ which is a contradiction.

Theorem 4.1.3. Let $\mathcal{B}, C, \mathcal{D}$ be bases of $R$. If $\mathcal{B}$ is congenial to $C$ and $C$ is congenial to $\mathcal{D}$ then $\mathcal{B}$ is congenial to $\mathcal{D}$.

Proof. Since $\mathcal{B}$ is congenial to $C$ then,$\forall c \in C$ the set $\mathcal{B}_{c}$ is finite. Also since $C$ is congenial to $\mathcal{D}$ then,$\forall d \in \mathcal{D}$ the set $C_{d}$ is finite. For fixed $d \in \mathcal{D}$, since each $\mathcal{B}_{c}$ and $C_{d}$ are finite then the union of all sets $B_{c}$ for all $c \in C_{d}$ is finite. From Lemma.4, $\forall d \in \mathcal{D}$ we have that $\mathcal{B}_{d} \subseteq \bigcup_{c \in C_{d}} \mathcal{B}_{c}$ which prove that the set $\mathcal{B}_{d}$ is finite. Therefore $\mathcal{B}$ is congenial to D. $\square$

\subsection{Isomorphic Modules Based on Different Bases}

The following theorem shows that if we have two bases of algebra, if one of these bases is amenable then the other basis is amenable under the condition that they are mutually congenital.

Lemma 4.2.1. For every $r \in R$ and $c_{0} \in C$, let $\mathfrak{U}=\bigcup_{b \in B_{c_{0}}} B_{b}$ then we have that ${ }_{r} C_{c_{0}} \subseteq \bigcup_{y \in \mathfrak{H}} C_{y}$.

Proof. Suppose $x \in{ }_{r} C_{c_{0}}$ and $x \notin \bigcup_{y \in \mathfrak{I}} C_{y}$ we show that this leads to a contradiction. So, if $x \in{ }_{r} C_{c_{0}}$ we get $(r x)_{c_{0}} \neq 0$. In addition, if $x \notin \bigcup_{y \in \mathfrak{H}} C_{y}$ we get $x \in \bigcap_{y \in \mathfrak{l}} C_{y}{ }^{c}=\{v \in C$ : $\left.(v)_{y}=0, \forall y \in \mathfrak{U}\right\}$. First since $B$ is a basis, we write $x$ as a linear combination of elements from $B, x=\sum_{y \in B}(x)_{y} y$. Then multiplying on the right by $r$ we get $r x=\sum_{y \in \mathcal{B}}(x)_{y}(r y)$. Now we write $r y$ as a linear combination of elements from $B$, we get

$$
\begin{aligned}
& r x=\sum_{b \in \mathcal{B}} \sum_{y \in B}(x)_{y}(r y)_{b} b \\
& \Rightarrow(r x)_{c_{0}}=\sum_{b \in \mathcal{B}} \sum_{y \in \mathcal{B}}(x)_{y}(r y)_{b}(b)_{c_{0}} .
\end{aligned}
$$

Now $(r x)_{c_{0}} \neq 0$ then $\exists y^{\prime}, b^{\prime} \in \mathcal{B}$ such that $(x)_{y^{\prime}} \neq 0,\left(r y^{\prime}\right)_{b^{\prime}} \neq 0$ and $\left(b^{\prime}\right)_{c_{0}} \neq 0$. Here if $\left(b^{\prime}\right)_{c_{0}} \neq 0$ then $b^{\prime} \in \mathcal{B}_{c_{0}}$ and if $\left(r y^{\prime}\right)_{b^{\prime}} \neq 0$ then $y \in \mathfrak{U}$, but $(x)_{y}=0$ for every $y \in \mathfrak{U}$ which is a contradiction. 
Theorem 4.2.2. Given two bases $\mathcal{B}$ and $C$. If $\mathcal{B}$ is amenable and $\mathcal{B}$ is congenial to $C$ and $\mathcal{C}$ is congenial to $\mathcal{B}$ then $\mathcal{C}$ is amenable.

Proof. Fix $r \in R$ and $c_{0} \in C$, it is sufficient to show that the set ${ }_{r} C_{c_{0}}$ contained in a finite set. From the previous Lemma we can see that ${ }_{r} \mathcal{C}_{c_{0}} \subseteq \bigcup_{y \in \mathfrak{l}} \mathcal{C}_{y}$. Now, Since $\mathcal{B}$ is congenial to $\mathcal{C}$, we have $\left|\mathcal{B}_{c_{0}}\right|<\infty$. Also, since $\mathcal{B}$ is amenable, for $r \in R$ and,$\forall b \in \mathcal{B}_{c_{0}}$ we have $\left|{ }_{r} \mathcal{B}_{b}\right|<\infty$. Let $\mathfrak{U}=\bigcup_{b \in \mathcal{B} r} \mathcal{B}_{b}$ we can see that the set $\mathfrak{U}$ is finite. Now Since $C$ is congenial to $\mathcal{B}, \forall y \in \mathfrak{U}$ we have $\left|C_{y}\right|<\infty$. Therefore the set $\bigcup_{y \in \mathfrak{l l}} C_{y}$ is finite.

In the previous theorem, we had that if we have two mutually congenial bases and one of them is amenable basis, then so is the other one. The examples below show that if we just have congeniality in either direction, are cannot predict neither amenability nor its absence for the one.

Example 4.2.1. Let $\mathcal{B}=\left\{x^{i}: i \geq 0\right\}$ and $\mathcal{E}=\{1\} \cup\left\{x^{i}+(-1)^{i+1} x^{i+1}: i \geq 0\right\}$ be two bases of $\mathbb{F}[x]$. Clearly $\mathcal{B}$ is amenable and $\mathcal{E}$ is congenial to $\mathcal{B}$ but yet $\mathcal{E}$ is not amenable. Thus in order to have $\mathcal{E}$ to be amenable we must have condition that $\mathcal{B}$ is congenial to $\mathcal{E}$.

Example 4.2.2. Let $\mathcal{B}=\left\{x^{i}: i \geq 0\right\}$ and $\mathcal{D}=\left\{\sum_{i=0}^{n} x^{i}: n \geq 0\right\}$ be two bases of $\mathbb{F}[x]$. Clearly $\mathcal{B}$ is amenable and $\mathcal{B}$ is congenial to $\mathcal{D}$ but yet $\mathcal{D}$ is not amenable. Thus in order to have $\mathcal{D}$ to be amenable we must have condition that $\mathcal{D}$ is congenial to $\mathcal{B}$.

Remark: we deal with infinite linear systems $A \cdot \alpha=\beta$ where A is row-finite matrix as it appears in [12]. Let $\mathrm{A}$ be matrix and let $\mathrm{H}$ be a matrix constructed by the infinite Gauss-Jordan elimination algorithm. Let also Q be the nonsingular matrix representing the composite of elementary row operators transforming A to $\mathrm{H}$ i.e. $Q \cdot A=H$. Multiplying both sides of $A \cdot \alpha=\beta$ by $Q$ we have: $A \cdot \alpha=\beta \Rightarrow H \cdot \alpha=Q \cdot \beta$ As $Q$ is nonsingular the converse statement also holds by multiplying both sides by $Q^{-1}$ of $H \cdot \alpha=Q \cdot \beta$ thus: $A \cdot \alpha=\beta \Leftrightarrow H \cdot y=Q \cdot \beta$. Meaning that any solution of $A \cdot \alpha=\beta$ is a solution of $H \cdot y=Q \cdot \beta$ and vice versa. For more details see [12]. 
Theorem 4.2.3. If $\mathcal{B}$ is congenial to $C$ then there exists an onto linear map $\phi: K^{\mathcal{B}} \rightarrow K^{C}$. If, In addition, $\mathcal{B}$ and $\mathcal{C}$ are amenable bases then $\phi$ is an $R-$ linear i.e. $\phi: \mathcal{M}_{\mathcal{B}} \rightarrow \mathcal{M}_{C}$ is epimorphism.

Proof. The claim is to show that $\phi$ is onto. Suppose that $\mathcal{B}$ is congenial to $\mathcal{C}$, that means $\forall c \in C \rightarrow\left|\left\{b \in \mathcal{B}:(b)_{c} \neq 0\right\}\right|<\infty$. Suppose that $\phi: M_{\mathcal{B}} \rightarrow \mathcal{M}_{C}$ is a map given by $\sum_{b \in \mathcal{B}} \alpha_{b} b \rightarrow \sum_{c \in C} \beta_{c} c$. We start with an arbitrary element, say $\sum_{c \in C} \beta_{c} c \in \mathcal{M}_{C}$ and write $\beta_{c}=\sum \alpha_{b}(b)_{c}$. We show that $\phi$ is homomorphism.

We show the map is onto by showing that the system $A \alpha=\beta$ has a solution where $\alpha$ is the representative column of the solution $\left(\alpha_{1}, \alpha_{2}, \ldots, \alpha_{n_{1}}, \ldots\right)$. So the goal is to find the $\alpha_{b}{ }^{\prime} s$.

Let $A=\left[I_{V}\right]_{\mathcal{B}}^{C}$ be the change of coordinate row-column finite matrix that changes $\mathcal{C}$-coordinates to $\mathcal{B}$-coordinates.

Represent the system in the matrix form as

$$
A \cdot\left(\begin{array}{c}
\alpha_{1} \\
\alpha_{2} \\
\vdots \\
\alpha_{n_{1}} \\
\vdots
\end{array}\right)=\left(\begin{array}{c}
\beta_{1} \\
\beta_{2} \\
\vdots \\
\beta_{n_{1}} \\
\vdots
\end{array}\right)
$$

Reorder the columns in $A$ so we get,

$$
\left(\begin{array}{cccccccc}
a_{11} & \cdots & a_{1 n_{1}} & 0 & \cdots & \cdots & \cdots & \\
a_{21} & \cdots & \cdots & \cdots & a_{2 n_{2}} & 0 & \cdots & \cdots \\
& \vdots & \vdots & \vdots & \vdots & \vdots & \vdots &
\end{array}\right) \cdot\left(\begin{array}{c}
\alpha_{1} \\
\vdots \\
\alpha_{n_{1}} \\
\vdots
\end{array}\right)=\left(\begin{array}{c}
\beta_{1} \\
\vdots \\
\beta_{n_{1}} \\
\vdots
\end{array}\right)
$$

Case 1. Without loss of generality we assume that $n_{i}<n_{j}$ if $i<j$. Multiplying the first row of the matrix $A$ by the $\alpha$ column, we get $a_{11} \alpha_{1}+\cdots+a_{1 n_{1}} \alpha_{n_{1}}=\beta_{1}$. Choose $\alpha_{i}=0$ for $1<i<n-1$ thus $\alpha_{n_{1}}=a_{1 n_{1}}{ }^{-1} \beta_{1}$. Now take $\alpha_{i}=0$ for $n_{1}<i<n_{2}$. Then we have $a_{2 n_{1}} \alpha_{n_{1}}+a_{2 n_{2}} \alpha_{n_{2}}=\beta_{2}$ therefore $\alpha_{n_{2}}=a_{2 n_{1}}{ }^{-1}\left(\beta_{2}-a_{2 n_{1}} \alpha_{n_{1}}\right)$. By continuing in this process 
we can find $\alpha$ which satisfies the system. So we proved the lemma when the inequality is strictly less $n_{i}<n_{j}$.

Case 2. If $n_{i}=n_{i+1}=\cdots=n_{i+t_{1}}$ for some $i$. Let $t_{1}$ to be the largest.

First let $i=1$ then we have

Now let

$$
\left(\begin{array}{ccc|cc}
a_{11} & \cdots & a_{1 n_{1}} & 0 & \cdots \\
a_{21} & \cdots & a_{2 n_{1}} & 0 & \cdots \\
\vdots & \vdots & \vdots & \vdots & \vdots \\
a_{t_{1} 1} & \cdots & a_{t_{1} n_{1}} & 0 & \cdots \\
\hline & B_{2} & & A_{2}
\end{array}\right) \cdot\left(\begin{array}{c}
\alpha_{1} \\
\vdots \\
\alpha_{n_{1}} \\
\vdots
\end{array}\right)=\left(\begin{array}{c}
\beta_{1} \\
\vdots \\
\beta_{n_{1}} \\
\vdots
\end{array}\right)
$$

$$
A_{1}=\left(\begin{array}{ccc}
a_{11} & \cdots & a_{1 n_{1}} \\
a_{21} & \cdots & a_{2 n_{1}} \\
\vdots & \vdots & \vdots \\
a_{t_{1} 1} & \cdots & a_{t_{1} n_{1}}
\end{array}\right)
$$

Now we want to show that the map $\psi=l_{A_{1}}: F^{n_{1}} \rightarrow F^{t_{1}}$ (left multiplication by $A_{1}$ ) is onto. Since the rows of $A_{1}$ are linearly independent then $t_{1} \leq n_{1}$ then the nullity of $\psi$ is equal to $n_{1}-t_{1}$. We know that $\operatorname{nullity}(\psi)+\operatorname{rank}(\psi)=\operatorname{dim}\left(F^{n_{1}}\right)$ this gives us $\left(n_{1}-t_{1}\right)+\operatorname{rank}(\psi)=n_{1}$, therefore $\operatorname{rank}(\psi)$ is equal to $t_{1}$. Since $\psi$ is onto we can find $\left(\alpha_{1}, \alpha_{2}, \ldots, \alpha_{n_{1}}\right)$. So Let $\Gamma_{1}=\left(\alpha_{1}, \alpha_{2}, \ldots, \alpha_{n_{1}}\right)$ and $\Omega_{2}=\left(\beta_{1}, \beta_{2}, \ldots, \beta_{n_{1}}\right)$. Then $A_{1} \Gamma_{1}=\Omega_{1} \Rightarrow \Gamma_{1}=A_{1}^{-1} \Omega_{1}$.

Next let $\Gamma_{2}=\left(\alpha_{n_{1}+1}, \ldots, \alpha_{n_{2}}\right)$ and $\Omega_{2}=\left(\beta_{t_{1}+1}, \ldots, \beta_{t_{1}+t_{2}}\right)$.

Then we have 


$$
\left(\begin{array}{cccc}
A_{1} & O & \ldots & \ldots \\
B_{2} & A_{2} & O & \ldots \\
\vdots & \vdots & \vdots & \vdots
\end{array}\right) \cdot\left(\begin{array}{c}
\Gamma_{1} \\
\alpha_{n_{1}+1} \\
\vdots \\
\alpha_{n_{2}} \\
\vdots
\end{array}\right)=\left(\begin{array}{c}
\beta_{1} \\
\vdots \\
\beta_{t_{1}} \\
\beta_{t_{1}+1} \\
\vdots \\
\beta_{t_{1}+t_{2}} \\
\vdots
\end{array}\right)
$$

Therefore $B_{2} \Gamma_{1}+A_{2} \Gamma_{2}=\Omega_{2} \Rightarrow A_{2} \Gamma_{2}=\Omega_{2}-B_{2} \Gamma_{1}$. To find $\Gamma_{2}$ we have to show that the map of left multiplication by $A_{2}$ is onto. Suppose that the map is not onto then

$$
\left(\begin{array}{cccc}
A_{1} & O & \cdots & \cdots \\
B_{2} & A_{2} & O & \cdots \\
\vdots & \vdots & \vdots & \vdots
\end{array}\right) \Rightarrow\left(\begin{array}{cccc}
A_{1} & O & \ldots & \ldots \\
B_{21} & A_{2}^{*} & O & \ldots \\
B_{22} & O & O & \ldots \\
\vdots & \vdots & \vdots & \vdots
\end{array}\right) \Rightarrow\left(\begin{array}{cccc}
A_{1} & O & \ldots & \ldots \\
B_{22} & O & O & \ldots \\
B_{21} & A_{2}^{*} & O & \ldots \\
\vdots & \vdots & \vdots & \vdots
\end{array}\right)
$$

Which is a contradiction since we chose $t_{1}$ to be large. Hence the map of left multiplication by $A_{2}$ is onto and $\Gamma_{2}=A_{2}^{-1} \Omega_{2}-A_{2}^{-1} B_{2} \Gamma_{1}$. Continue in this process we can find a solution $\alpha$ for the system.

Theorem 4.2.4. Suppose that $\mathcal{B}$ and $C$ are two amenable bases of $R$. if $\mathcal{B}$ and $C$ are mutually congenial then $\mathcal{M}_{\mathcal{B}} \cong \mathcal{M}_{C}$ naturally.

Proof. Suppose that $\mathcal{B}$ and $C$ are mutually congenial and induced an $R$ - linear maps $\Phi_{\mathcal{B C}}: \mathcal{M}_{\mathcal{B}} \rightarrow \mathcal{M}_{C}$ and $\Phi_{C B}: \mathcal{M}_{C} \rightarrow \mathcal{M}_{\mathcal{B}}$ we show that these two maps are mutually inverse. First we show that $\Phi_{\mathcal{B C}}$ is a $R$-homomorphism.

Let $\mu: \mathcal{B} \rightarrow K$ and $\sigma: C \rightarrow K$ where $\mu \in \mathcal{M}_{\mathcal{B}}$ and $\sigma \in \mathcal{M}_{C}$. $\Phi_{\mathcal{B C}}$ is defined by $\Phi_{\mathcal{B} C}(\mu)(c)=\sum_{b \in \mathcal{B}}(b)_{c} \mu(b)$.

Now we have,

- $\Phi_{\mathcal{B C}}\left(\mu_{1}+\mu_{2}\right)(c)=\sum_{b \in \mathcal{B}}(b)_{c}\left(\mu_{1}+\mu_{2}\right)(b)$

$$
=\sum_{b \in \mathcal{B}}(b)_{c} \mu_{1}(b)+\sum_{b \in \mathcal{B}}(b)_{c} \mu_{2}(b)
$$


$=\Phi_{\mathcal{B C}}\left(\mu_{1}\right)(c)+\Phi_{\mathcal{B C}}\left(\mu_{2}\right)(c)$.

- $\Phi_{\mathcal{B C}}(r \cdot \mu)(c)=\sum_{b \in \mathcal{B}}(b)_{c}(r \mu)(b)$

$=r \cdot \sum_{b \in \mathcal{B}}(b)_{c} \mu(b)$

$=r \cdot \Phi_{\mathcal{B} C}(\mu)(c)$.

Therefore $\Phi_{\mathcal{B} C}$ is an $R$-homomorphism, similarly $\Phi_{C B}$ is homomorphism.

Now we want show that $\Phi_{\mathcal{B C}}$ is isomorphism, we claim that $\Phi_{C B} \circ \Phi_{\mathcal{B C}}=1$.

$\Phi_{C B} \circ \Phi_{\mathcal{B C}}(\mu)(b)=\Phi_{C B}\left(\Phi_{\mathcal{B} C}(\mu)\right)(b)$

$=\sum_{c \in C}(c)_{b} \Phi_{\mathcal{B C}}(\mu)(c)$

$=\sum_{c \in C}(c)_{b} \sum_{a \in \mathcal{B}}(a)_{c} \mu(a)$

$=\sum_{c \in C} \sum_{a \in \mathcal{B}}(c)_{b}(a)_{c} \mu(a)$

$=\sum_{a \in \mathcal{B}}\left(\sum_{c \in C}(a)_{c}(c)_{b}\right) \mu(a)$

$=\sum_{a \in \mathcal{B}}(a)_{b} \mu(a) \quad$, Since $\sum_{c \in C}(a)_{c}(c)_{b}=(a)_{b}=\mu(b)$

Since $\Rightarrow(a)_{b}= \begin{cases}1 & \text { if } a=b \\ 0 & \text { if } a \neq b\end{cases}$

Similarly $\Phi_{\mathcal{B C}} \circ \Phi_{C B}=1 \Rightarrow \Phi_{\mathcal{B} C}$ is isomorphism. Therefore $\Phi_{\mathcal{B} C}, \Phi_{C B}$ are mutually inverse.

As an application of our main result we have these nice examples.

Example 4.2.3. Let $R=\mathbb{F}[x]$ and $\mathcal{B}=\left\{x^{i}: i \geq 0\right\}$ be the standard basis of $R$.

$\forall n \geq 0$ and let $\mathcal{G}=\left\{\begin{array}{cr}x^{i} & \text { if } i=2 n \\ x^{i}+x^{i+1} & \text { if } i=2 n+1\end{array}\right.$

Clearly $\mathcal{B}$ and $\mathcal{G}$ are mutually congenial then by Theorem 4.2.3 $\mathcal{M}_{\mathcal{B}}$ and $\mathcal{M}_{\mathcal{G}}$ are naturally isomorphic.

Example 4.2.4. Let $\mathcal{B}=\left\{x^{i}: i \geq 0\right\}$ and let $C=\{1\} \cup\left\{x^{i}+x^{i+1}: i \geq 0\right\}$ be bases of $\mathbb{F}[x]$

. These two bases are amenable we can see that $\mathcal{C}$ is congenial to $\mathcal{B}$ and induced a map $\phi: \mathcal{M}_{C} \rightarrow \mathcal{M}_{\mathcal{B}}$. Since $\mathcal{B}$ is not congenial to $\mathcal{C}, \phi$ is not one to one. 
Let $P_{0}=1$

$P_{i}=x^{i-1}(1+x), i \geq 0$

and $\phi\left(\sum_{i=0}^{n}(-1)^{i} P_{i}\right)=(-1)^{n} x^{n}$. We show that $\phi\left(\sum_{i=0}^{\infty}(-1)^{i} P_{i}\right)=0$. Let $a=\sum_{i=0}^{\infty}(-1)^{i} P_{i}$.

Then we have,

$$
\begin{aligned}
& a=\sum_{i=0}^{n}(-1)^{i} P_{i}+(-1)^{n} x^{n}(a-1) \\
& \Rightarrow \phi(a)=\phi\left(\sum_{i=0}^{n}(-1)^{i} P_{i}\right)+(-1)^{n} x^{n}(\phi(a)-1) \\
& \Rightarrow \phi(a)=(-1)^{n} x^{n}+(-1)^{n} x^{n}(\phi(a)-1)=(-1)^{n} x^{n}+(-1)^{n} x^{n} \phi(a)-(-1)^{n} x^{n}=(-1)^{n} x^{n} \phi(a) \\
& \Rightarrow \phi(a)\left(1-(-1)^{n} x^{n}\right)=0 \\
& \Rightarrow \phi(a)=0
\end{aligned}
$$

Therefore $\phi$ is not one to one since $\phi(0)=0$.

Definition 4.2.1. Let $\mathcal{B}$ and $\mathcal{C}$ be bases. Let $\mathcal{B}$ be congenial to the $\mathcal{C}$ then the matrix representation relative to $(\mathcal{B}, C), A=\left[I_{R}\right]_{\mathcal{B}}^{C}$ is called a congeniality matrix. Furthermore, if $C$ not congenial to $\mathcal{B}$, then the matrix representation relative to $(\mathcal{B}, C),\left[I_{R}\right]_{\mathcal{B}}^{C}=A$ is called a proper congeniality matrix.

Definition 4.2.2. A Module $\mathcal{M}$ over infinite dimensional algebra $R$ is called a basic module if there exists a basis $\mathcal{B}$ such that $\mathcal{M}=\mathcal{M}_{\mathcal{B}}$.

Definition 4.2.3. Let $R$ be an infinite dimensional algebra and $\mathcal{B}=\mathcal{B}_{0}$ be an amenable basis and let $A$ any proper congeniality matrix. Then we can produce a series

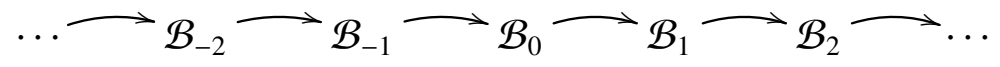

Where for every $i, \mathcal{B}_{i}$ is congenial to $\mathcal{B}_{i+1}$ and $A=\left[I_{R}\right]_{B_{i}}^{B_{i+1}}$. We call that series an A-series. Now, If there exists $n \in \mathbb{Z}^{+}$such that $j \geq n, \mathcal{B}_{j}$ is not amenable. A smallest such $n$ yield $B_{n}$ is the last amenable basis in the A-series. We say that $\mathcal{M}_{\mathcal{B}_{n}}$ is an A-simple basic module.

\section{Example 4.2.5. Let}




$$
A=\left(\begin{array}{cccccccc}
1 & 1 & 0 & 0 & 0 & \cdots & 0 & \cdots \\
0 & 1 & 1 & 0 & 0 & \cdots & 0 & \cdots \\
0 & 0 & 1 & 1 & 0 & \cdots & 0 & \cdots \\
0 & 0 & 0 & 1 & 1 & \cdots & 0 & \cdots \\
\vdots & \vdots & \vdots & \vdots & \vdots & \vdots & \vdots &
\end{array}\right)
$$

Let $\mathcal{B}_{0}=\left\{x^{i}: i \geq 0\right\}$ we can produced a series from $A$ and $B_{0}$

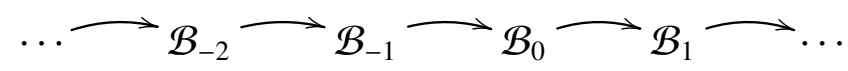

Where $\mathcal{B}_{-1}=\{1\} \cup\left\{x^{i}+x^{i+1}: i \geq 0\right\}, \mathcal{B}_{-2}=\{1, x+1\} \cup\left\{x^{i}+2 x^{i+1}+x^{i+2}: i \geq 0\right\}$ and $\mathcal{B}_{1}=\left\{\sum_{i=0}^{n}(-1)^{n-i} x^{i}: n \geq 0\right\}$. The bases $\mathcal{B}_{-2}, \mathcal{B}_{-1}$ and $\mathcal{B}_{0}$ are amenable, however $\mathcal{B}_{1}$ is not amenable. In fact $\mathcal{B}_{n}$ is not amenable for any $n \geq 1$ and $\mathcal{M}_{\mathcal{B}_{0}}$ is an A-simple basic module.

For the algebra of the polynomials, there could be a series that non of the bases are amenable. Furthermore, it turns out that there is a series that is if one basis is amenable in one stage doesn't imply that it remain amenable basis in the next stage. Also it doesn't imply that the basis before is amenable. As we see in the following examples.

Example 4.2.6. Let

$$
A=\left(\begin{array}{cccccccc}
1 & 1 & 0 & 0 & 0 & \cdots & 0 & \cdots \\
0 & -1 & 1 & 0 & 0 & \cdots & 0 & \cdots \\
0 & 0 & 1 & 1 & 0 & \cdots & 0 & \cdots \\
0 & 0 & 0 & -1 & 1 & \cdots & 0 & \cdots \\
\vdots & \vdots & \vdots & \vdots & \vdots & \vdots & \vdots &
\end{array}\right)
$$

Let $\mathcal{B}_{0}=\left\{x^{i}: i \geq 0\right\}$ we can produced a series from $A$ and $B_{0}$



Where $\mathcal{B}_{-1}=\left\{\sum_{i=0}^{n}(-1)^{i} x^{i}: n \geq 0\right\}, \mathcal{B}_{-2}=\{1\} \cup\left\{x^{i}+x^{i+2}: i \geq 0\right\}$ and $\mathcal{B}_{1}=$ $\left\{1,1-x,-1+x+x^{2},-1+x+x^{2}-x^{3}, 1-x-x^{2}+x^{3}+x^{4}, 1-x-x^{2}+x^{3}+x^{4}-x^{5}, \cdots\right\}$. The bases $\mathcal{B}_{-2}, \mathcal{B}_{0}$ are amenable, however $\mathcal{B}_{-1}$ and $\mathcal{B}_{1}$ are not amenable. 
In the example below, we can produce an A-series where all bases are not amenable.

\section{Example 4.2.7. Let}

$$
A=\left(\begin{array}{cccccccc}
1 & 1 & 0 & 0 & 0 & \cdots & 0 & \cdots \\
0 & 1 & 1 & 0 & 0 & \cdots & 0 & \cdots \\
0 & 0 & 1 & 1 & 0 & \cdots & 0 & \cdots \\
0 & 0 & 0 & 1 & 1 & \cdots & 0 & \cdots \\
\vdots & \vdots & \vdots & \vdots & \vdots & \vdots & \vdots &
\end{array}\right)
$$

Let $\mathcal{B}_{0}=\left\{1+x^{i}: i \geq 0\right\}$ we can produced a series from $A$ and $B_{0}$

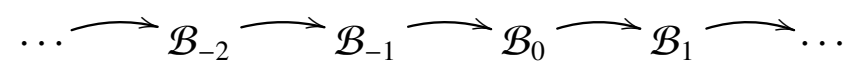

Where $\mathcal{B}_{-1}=\{1,2+x\} \cup\left\{2+x^{i}+x^{i+1}: i \geq 1\right\}, \mathcal{B}_{-2}=\{1,3+x\} \cup\left\{4+2 x^{i}+x^{i+1}: i \geq 1\right\}$, and $\mathcal{B}_{1}=\left\{1, x, 1-x+x^{2}, x-x^{2}+x^{3}, 1-x+x^{2}-x^{3}+x^{4}, x-x^{2}+x^{3}-x^{4}+x^{5}, \cdots\right\}$. The bases $\mathcal{B}_{-2}, \mathcal{B}_{-1}, \mathcal{B}_{0}$ and $\mathcal{B}_{1}$ all are not amenable. 


\section{Leavitt Path Algebra}

Now we introduce the notion of Leavitt Path Algebra as it appears in many papres, including [2], [14] and [1].

Definition 5.0.4. Let $K$ be a field and $E$ be a row-finite graph i.e. every vertex emits only finitely many edges. The path $K$-algebra over $E$ is defined as the free $K$-algebra $K\left[E^{0} \cup E^{1}\right]$ with the relations:

1. $v_{i} v_{j}=\delta_{i j} v_{i}$ for every $v_{i}, v_{j} \in E^{0}$.

2. $e_{i}=e_{i} r\left(e_{i}\right)=s\left(e_{i}\right) e_{i}$ for every $e_{i} \in E^{1}$.

This algebra is denoted by $A_{K}(E)$.

Definition 5.0.5. Given a graph $E$ we define the extended graph of $E$ as the new graph $\widehat{E}=\left(E^{0}, E^{1} \cup\left(E^{1}\right)^{*}, r^{\prime}, s^{\prime}\right)$ where $\left(E^{1}\right)^{*}=\left\{e_{i}^{*}: e_{i} \in E^{1}\right\}$ and the functions $r^{\prime}$ and $s^{\prime}$ are defined as

$$
\left.r^{\prime}\right|_{E^{1}}=r,\left.s^{\prime}\right|_{E^{1}}=s, r^{\prime}\left(e_{i}^{*}\right)=s\left(e_{i}\right) \text { and } s^{\prime}\left(e_{i}^{*}\right)=r\left(e_{i}\right) .
$$

The elements of $E^{1}$ are called (real) edges, while for $e \in E^{1}$ we call $e^{*}$ a ghost edge. The set $e^{*} \in E^{1}$ will be denoted by $\left(E^{1}\right)^{*}$. We let $r\left(e^{*}\right)$ denote $s(e)$, and we let $s\left(e^{*}\right)$ denote $r(e)$. If $\mu=e_{1} \ldots e_{n}$ is a path, then we denote by $\mu *$ the element $e_{n}^{*} \ldots e_{1}^{*}$ of $L_{K}(E)$.

Definition 5.0.6. For a field $K$ and a row-finite graph $E$, the Leavitt path $K$-algebra $L_{K}(E)$ is defined as the $K$-algebra generated by a set $\left\{v: v \in E^{0}\right\}$ of pairwise orthogonal idempotents, together with a set of variables $E_{1} \cup E_{1}^{*}$, which satisfy the following relations:

(V) $v v^{\prime}=\delta_{v, v^{\prime}} v$ for all $v, v^{\prime} \in E^{0}$,

(E1) $s(e) e=e r(e)=e$ for all $e \in E^{1}$

(E2) $r(e) e^{*}=e^{*} s(e)=e^{*}$ for all $e \in E^{1}$ 
(CK1) $e^{*} e^{\prime}=\delta_{e, e^{\prime}} r(e)$ for all $e, e^{\prime} \in E^{1}$

(CK2) $v=\sum_{e \in E^{1}: s(e)=v} e e^{*}$ for every $v \in E^{0}$ that emits edges.

The conditions CK1 and CK2 are called the Cuntz-Krieger relations. In particular condition CK2 is the Cuntz-Krieger relation at $v_{i}$. If $v_{i}$ is a sink, we do not have a CK2 relation at $v_{i}$. Note that the condition of row-finiteness is needed in order to define the equation CK2 .

Definition 5.0.7. A vertex $v$ in $E^{0}$ is a bifurcation (or there is a bifurcation at $v$ ) if $s^{-1}(v)$ has at least two elements.

The following are the well-known examples of Leavitt Path Algebras.

Example 5.0.8. Matrix algebras $M_{n}(K)$ : Consider the graph $E$ defined by $E^{0}=$ $v_{1}, \ldots, v_{n}, E 1=e_{1}, \ldots, e_{n-1}$ and $s\left(e_{i}\right)=v_{i}$ and $r\left(e_{i}\right)=v_{i+1}$ for $i=1, \ldots, n-1$. Then $L_{K}(E) \cong M_{n}(K)$, via the map $v_{i} \rightarrow e_{(i, i)}, e_{i} \rightarrow e e_{(i, i+1)}$, and $e_{i}^{*} \rightarrow e e_{(i+1, i)}$ (where $e_{(i, j)}$ denotes the standard $(i, j)$-matrix unit in $\left.M_{n}(K)\right)$.

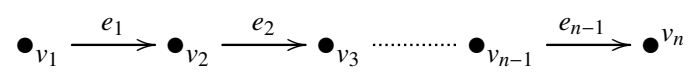

Example 5.0.9. Laurent polynomial algebras $K[x, x-1]$ : Consider the "one vertex, one loop"graph $E$ defined by $E^{0}=\{*\}, E^{1}=\{x\}$. Then clearly $L_{K}(E) \cong K[x, x-1]$.

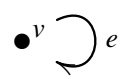


Example 5.0.10. Consider the rose with $n$ petals graph $E$ defined by $E^{0}=\{*\}, E_{1}=$ $y_{1}, \ldots, y_{n}($ where $n \geq 2)$. Then $L_{K}(E) \cong L_{K}(1, n)$.

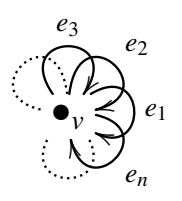

Lemma 5.0.5. [2] Every monomial in $L(E)$ is of the following form.

1. $k_{i} v_{i}$ with $k_{i} \in K$ and $v_{i} \in E^{0}$, or

2. $k e_{i_{1}} \cdots e_{i_{\sigma}} e_{j_{1}}^{*} \cdots e_{j_{\tau}}^{*}$ where $k \in K ; \sigma, \tau \geq 0, \sigma+\tau>0, e \in E^{1}$ and $e^{*} \in\left(E_{1}\right)^{*}$ for $0 \leq s \leq \sigma, 0 \leq t \leq \tau$.

In this section we introduce a family of bases for Leavitt Path Algebras which we will call standard and show that all standard bases are amenable.

Now we define a basis of Leavitt Path Algebra $L_{k}(E)$ over directed graphs as it appears in $[4]$.

For an arbitrary vertex $v$ which is not a sink, choose an edge $\phi(v)$ such that $s(\phi(v))=v$. We will refer to this edge as special. In other words, we fix a function $\phi: V \rightarrow E^{1}$ such that $s(\phi(v))=v$ for an arbitrary $v \in V$.

Definition 5.0.8. The union of the following sets form a basis of the Leavitt path algebra $L_{K}(E)$ denoted by $\mathcal{B}_{\phi}$.

- $\{v: v \in V\}$,

- $\{p: p$ is a path in $E\} \cup\left\{p^{*}: p\right.$ is a path in $\left.E\right\}$ 
- $\left\{p q^{*}: p=e_{1} \ldots e_{n}, q=f_{1} \ldots f_{m}, e_{i}, f_{j} \in E, r\left(e_{n}\right)=r\left(f_{m}\right)\right.$ and $p q^{*}$ is reduced, with the condition that the last edges $e_{n}$ and $f_{m}$ are either distinct or equal, but not special\}.

Let $\alpha, \beta, \gamma$ and $\delta$ be paths in $L_{k}(E)$, then

$\left(\alpha \beta^{*}\right)\left(\gamma \delta^{*}\right)=\left\{\begin{array}{cc}\alpha \gamma^{\prime} \delta^{*} & \text { if } \gamma=\beta \gamma^{\prime} \\ \alpha \beta^{\prime *} \delta^{*} & \text { if } \beta=\gamma \beta^{\prime} \\ 0 & \text { otherwise }\end{array}\right.$

Now we represent the product with respect to the basis $\mathcal{B}_{\phi}$.

$\left(\alpha \beta^{*}\right)\left(\gamma \delta^{*}\right)=\left\{\begin{array}{rr}\alpha \delta^{*} & \text { if } \gamma=\beta \text { \& end }(\alpha) \text { or } \operatorname{end}(\delta) \notin \mathcal{F} \\ & \text { or if end }(\alpha), \text { end }(\delta) \in \mathcal{F} \& s(\operatorname{end}(\alpha)) \neq s(\operatorname{end}(\delta)) \\ \sum \alpha^{\prime} \delta^{\prime *} & \text { if } \operatorname{end}(\alpha)=\operatorname{end}(\delta) \in \mathcal{F}\end{array}\right.$

For all $\alpha^{\prime}, \beta^{\prime}, \gamma^{\prime}$ and $\delta^{\prime}$ in $L_{k}(E)$.

The following notions and terminology were introduced in [6].

Definition 5.0.9. For a path $\alpha=\alpha_{1} \ldots \alpha_{k}$ and for $1 \leq l \leq k$, denote by $(\alpha)_{\tau \leq l}=\alpha_{1} \ldots \alpha_{l}$ and $(\alpha)_{\tau>l}=\alpha_{l+1} \ldots \alpha_{k}$ the truncation at $l$.

Definition 5.0.10. Two paths $\alpha$ and $\beta$ are tail-equivalent if there exists $n$ and $m$ such that $(\alpha)_{\tau>n}=(\beta)_{\tau>m}$

In the Theorem 1.6.5. below we show that $\mathcal{B}$ is amenable. Before that we prove some useful Lemmas.

Lemma 5.0.6. Let $L_{k}(E)$ be a Leavitt path algebra and $\mathcal{B}$ be its standard basis induced by $\phi: V \rightarrow E^{1}$. Let $\alpha, \delta, \varphi$ and $\varepsilon$ be paths in $L_{K}(E)$ with $\alpha=\alpha_{1} \ldots \alpha_{k}, \beta=\beta_{1} \ldots \beta_{l}, \varepsilon=\varepsilon_{1} \ldots \varepsilon_{m}$ and $\varphi=\varphi_{1} \ldots \varphi_{p}$. For every $\alpha \beta^{*}, \varepsilon \varphi^{*} \in \mathcal{B}$, if $\alpha=\varepsilon$ and $\beta$ and $\varphi$ are tail-equivalent then $w e$ have $\left|\left\{\gamma \delta^{*} \in \mathcal{B}:\left(\alpha \beta^{*} \gamma \delta^{*}\right)_{\varepsilon \varphi^{*}} \neq 0\right\}\right|=n+1$, where $n$ is the number of the edges in the commen tail of the paths $\varphi$ and $\beta$.

Proof. We prove by induction on $n$ that there are $n+1$ ways of $\gamma \delta^{*}$ such that $\left(\alpha \beta^{*} \gamma \delta^{*}\right)_{\varepsilon \varphi^{*}} \neq 0$ 
- We prove the statement when $n=1$. If $n=1$ then $\beta_{l}=\varphi_{p}$. We have two ways such that $\left(\alpha \beta^{*} \gamma \delta^{*}\right)_{\alpha \varphi^{*}} \neq 0$ :

1. $\left(\alpha \beta^{*}\left(\beta \varphi^{*}\right)\right)_{\alpha \varphi^{*}}=\left(\alpha \varphi^{*}\right)_{\alpha \varphi^{*}} \neq 0$.

2. $\left(\alpha \beta^{*}\left(\beta_{\tau \leq l-1} \varphi_{\tau \leq p-1}^{*}\right)\right)_{\alpha \varphi^{*}}=\left(\alpha \beta_{l}^{*} \varphi_{\tau \leq p-1}^{*}\right)_{\alpha \varphi^{*}}=\left(\alpha \varphi_{p}^{*} \varphi_{\tau \leq p-1}^{*}\right)_{\alpha \varphi^{*}}=\left(\alpha \varphi^{*}\right)_{\alpha \varphi^{*}} \neq 0$.

When $n=2$ then $\beta_{l-1} \beta_{l}=\varphi_{p-1} \varphi_{p}$.

We have 3 ways such that

$\left(\alpha \beta^{*} \gamma \delta^{*}\right)_{\alpha \varphi^{*}} \neq 0$

1. $\left(\alpha \beta^{*}\left(\beta \varphi^{*}\right)\right)_{\alpha \varphi^{*}}=\left(\alpha \varphi^{*}\right)_{\alpha \mu^{*}} \neq 0$.

2. $\left(\alpha \beta^{*}\left(\beta_{\tau \leq l-1} \varphi_{\tau \leq p-1}^{*}\right)\right)_{\alpha \varphi^{*}}=\left(\alpha \beta_{l}^{*} \varphi_{\tau \leq p-1}^{*}\right)_{\alpha \varphi^{*}}=\left(\alpha \varphi^{*}\right)_{\alpha \varphi^{*}} \neq 0$.

3. $\left(\alpha \beta^{*}\left(\beta_{\tau \leq l-2} \varphi_{\tau \leq p-2}^{*}\right)\right)_{\alpha \mu^{*}}=\left(\alpha \beta_{l}^{*} \beta_{l-1}^{*} \varphi_{\tau \leq p-2}^{*}\right)_{\alpha \varphi^{*}}=\left(\alpha \varphi^{*}\right)_{\alpha \varphi^{*}} \neq 0$.

- Suppose the argument is true for $n$.

- Now, we show that the argument is true for $n+1$. When we have $n+1$ then $\beta_{\tau>l-(n+1)}=$ $\varphi_{\tau>p-(n+1)}$. Since $\beta_{l-n-1} \beta_{\tau>l-(n)}=\varphi_{p-n-1} \varphi_{\tau>p-n}$ then we have $\beta_{\tau>l-(n)}=\varphi_{\tau>p-n}$, by our assumption there are $n+1$ ways such that $\left(\alpha \beta^{*} \gamma \delta^{*}\right)_{\alpha \varphi^{*}} \neq 0$. Also we have

$$
\begin{aligned}
& \left(\alpha \beta^{*}\left(\beta_{\tau \leq l-n-1} \varphi_{\tau \leq l-n-1}^{*}\right)\right)_{\alpha \varphi^{*}} \\
& =\left(\alpha \beta_{\tau>l-n-1}^{*} \varphi_{\tau \leq p-n-1}^{*}\right)_{\alpha \mu^{*}} \\
& =\left(\alpha \varphi^{*}\right)_{\alpha \varphi^{*}} \neq 0 .
\end{aligned}
$$

Thus we have $n+2$ ways. Therefore by induction the lemma holds

Lemma 5.0.7. For every $\alpha \beta^{*}, \varepsilon \varphi^{*} \in \mathcal{B}$, let $\varepsilon$ be initial subpath of $\alpha$ but the last term i.e. $\varepsilon=\alpha_{\tau<t} e_{t}$ where $1 \leq t \leq n$.If $\alpha_{i}$ is special where $t \leq i \leq n$ and $\varphi=\varphi^{\prime} e_{t}$, then we have one way such that $\left(\alpha \beta^{*} \gamma \delta^{*}\right)_{\alpha \varphi^{*}} \neq 0$. 


$$
\begin{aligned}
& \text { Proof. }\left(\alpha \beta^{*}\left(\beta \alpha_{n}^{*} \alpha_{n-1}^{*} \ldots \alpha_{t}^{*} \varphi^{* *}\right)\right)_{\varepsilon \mu^{*}}=\left(\alpha_{\tau<n}\left(\alpha_{n} \alpha_{n}^{*}\right) \alpha_{n-1}^{*} \ldots \alpha_{t}^{*} \varphi V^{*}\right)_{\varepsilon \mu^{*}} \\
& =\left(\alpha_{\tau<n-1}\left(\alpha_{n-1} \alpha_{n-1}^{*}\right) \alpha_{n-2} \ldots \alpha_{t}^{*} \varphi^{*}\right)_{\varepsilon \varphi^{*}}=\left(\alpha_{\tau<t}\left(\alpha_{t} \alpha_{t}^{*}\right) \varphi^{*}\right)_{\varepsilon \mu^{*}} \\
& =\left(\alpha_{\tau<t}\left(v-\sum_{s\left(e_{j}\right)=v} e_{j} e_{j}^{*}\right) \varphi^{*}\right)_{\varepsilon \varphi^{*}}=\left(\alpha_{\tau<t}\left(e_{t} e_{t}^{*}\right) \varphi^{*}\right)_{\varepsilon \varphi^{*}} \neq 0 . \square
\end{aligned}
$$

Lemma 5.0.8. For every $\alpha \beta^{*}, \varepsilon \varphi^{*} \in \mathcal{B}$, If $\varepsilon$ is initial subpath of $\alpha$ i.e. $\varepsilon=\alpha_{\tau<t}$ where $1 \leq t \leq n$ and $\alpha_{i}$ are special where $t \leq i \leq n$ then we have one way such that $\left(\alpha \beta^{*} \gamma \delta^{*}\right)_{\varepsilon \varphi^{*}}$.

$$
\begin{aligned}
& \text { Proof. }\left(\alpha \beta^{*}\left(\beta \alpha_{n}^{*} \alpha_{n-1}^{*} \ldots \alpha_{t}^{*} \phi^{*}\right)\right)_{\varepsilon \phi^{*}}=\left(\alpha_{\tau<n}\left(\alpha_{n} \alpha_{n}^{*}\right) \alpha_{n-1}^{*} \ldots \alpha_{t}^{*} \phi^{*}\right)_{\varepsilon \phi^{*}} \\
& =\left(\alpha_{\tau<n-1}\left(\alpha_{n-1} \alpha_{n-1}^{*}\right) \alpha_{n-2} \ldots \alpha_{t}^{*} \phi^{*}\right)_{\varepsilon \phi^{*}}=\left(\alpha_{\tau<t}\left(\alpha_{t} \alpha_{t}^{*}\right) \phi^{*}\right)_{\varepsilon \phi^{*}} \\
& =\left(\alpha_{\tau<t}\left(v-\sum_{s\left(e_{j}\right)=v} e_{j} e_{j}^{*}\right) \phi^{*}\right)_{\varepsilon \phi^{*}}=\left(\alpha_{\tau<t} \phi^{*}\right)_{\varepsilon \phi^{*}} \neq 0 .
\end{aligned}
$$

Theorem 5.0.9. Let $\mathcal{B}$ be a basis of Leavitt path algebra $L_{k}(E)$ over directed graphs that has bifurcations as describe above. Then $\mathcal{B}$ is amenable.

Proof. There are two cases $|\varepsilon|>|\alpha|$ and $|\varepsilon| \leq|\alpha|$ to be considered.

Case 1. $|\varepsilon|>|\alpha|$

Since $|\varepsilon|>|\alpha|$ we need to extend the path $\alpha$. From the definition above we have $\left(\alpha \beta^{*}\right)\left(\gamma \delta^{*}\right) \neq 0 \Leftrightarrow \gamma=\beta \gamma_{1}$. Therefore $\left(\alpha \beta^{*}\right)\left(\gamma \delta^{*}\right)=\left(\alpha \beta^{*}\right)\left(\beta \gamma_{1} \delta^{*}\right)=\alpha \gamma_{1} \delta^{*}$.

Now, fix $\alpha \beta^{*}$ and $\varepsilon \varphi^{*}$ since $|\varepsilon|>|\alpha|$ then $\alpha$ is initial subpath of $\varepsilon$ i.e. if $\alpha=\alpha_{1} \ldots \alpha_{n}$ and $\varepsilon=\varepsilon_{1} \ldots \varepsilon_{m}$ then $\varepsilon=\alpha \varepsilon_{\tau>n}$. Therefore the only way to get $\varepsilon \varphi^{*}$ is to multiply from the right $\alpha \beta^{*}$ by $\beta \varepsilon_{\tau>n} \varphi^{*}$.

Case 2. $|\varepsilon| \leq|\alpha|$

Since $|\varepsilon| \leq|\alpha|$ we have two sub-cases:

Case 2.1. When $\alpha=\varepsilon$.

If $\beta$ and $\varphi$ are not tail-equivalent then we have only one way so that $\left(\alpha \beta^{*} \gamma \delta^{*}\right)_{\varepsilon \varphi^{*}} \neq 0$. Which is $\left(\alpha \beta^{*} \beta \varphi^{*}\right)_{\varepsilon \varphi^{*}}=\left(\alpha \varphi^{*}\right)_{\varepsilon \phi^{*}} \neq 0$

Now if $\beta$ and $\varphi$ are tail-equivalent then see Lemma 5.0.6. 
Case 2.2. When $\alpha \neq \varepsilon$.

\section{Lemma 5.0.7 and Lemma 5.0.8}

Example 5.0.11. Let $R=L_{K}(E)$ be Leavitt path algebra over graph E. Let $\mathcal{B}_{e}$ and $\mathcal{B}_{f}$ be two bases of $R$ where $\mathcal{B}_{e}=\left\{\alpha \beta^{*}: \alpha \beta^{*} \neq \alpha^{\prime} e e^{*} \beta^{\prime *}\right\}$ and $\mathcal{B}_{f}=\left\{\alpha \beta^{*}: \alpha \beta^{*} \neq \alpha^{\prime} f f^{*} \beta^{* *}\right\}$. We have that $\mathcal{B}_{e}$ and $\mathcal{B}_{f}$ are mutually congenial then by Theorem 4.2.3 $\mathcal{M}_{\mathcal{B}_{e}}, \mathcal{M}_{\mathcal{B}_{f}}$ are naturally isomorphic.

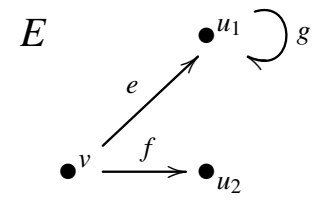

Example 5.0.12. Let $R=L_{K}(F)$ be Leavitt path algebra over graph $F$. Let $\mathcal{B}_{e}$ and $\mathcal{B}_{f}$ be two bases of $R$ where $\mathcal{B}_{e}=\left\{\alpha \beta^{*}: \alpha \beta^{*} \neq \alpha^{\prime} e e^{*}{\beta^{*}}^{*}\right.$ and $\mathcal{B}_{f}=\left\{\alpha \beta^{*}: \alpha \beta^{*} \neq \alpha^{\prime} f f^{*} \beta^{* *}\right\}$. We have that $\mathcal{B}_{f}$ is congenial to $\mathcal{B}_{e}$ but $\mathcal{B}_{e}$ is not congenial to $\mathcal{B}_{f}$. Therefore, the $R-$ linear map $\psi: \mathcal{M}_{\mathcal{B}_{e}} \rightarrow \mathcal{M}_{\mathcal{B}_{f}}$ is an epimorphism.

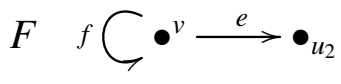

Example 5.0.13. Let $R=L_{K}(G)$ be Leavitt path algebra over graph $G$. Let $\mathcal{B}_{e}$ and $\mathcal{B}_{f}$ be two bases of $R$ where $\mathcal{B}_{e}=\left\{\alpha \beta^{*}: \alpha \beta^{*} \neq \alpha^{\prime} e e^{*} \beta^{*}\right\}$ and $\mathcal{B}_{f}=\left\{\alpha \beta^{*}: \alpha \beta^{*} \neq \alpha^{\prime} f f^{*} \beta^{*}\right\}$. We 
have that $\mathcal{B}_{f}$ is not congenial to $\mathcal{B}_{e}$ and $\mathcal{B}_{e}$ is not congenial to $\mathcal{B}_{f}$. Therefore, the $R$-linear map $\xi: \mathcal{M}_{\mathcal{B}_{e}} \rightarrow \mathcal{M}_{\mathcal{B}_{f}}$ is not a bijection map.

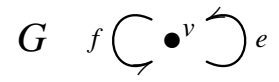




\section{Future Work}

In this section, we list some open problems related to our topic.

1. Let $\mathcal{B}, C$ be amenable bases of $R$. If $\mathcal{B}$ is congenial to $C$ and induced a map $\phi: \mathcal{M}_{\mathcal{B}} \rightarrow \mathcal{M}_{C}$ such that $\phi$ is one to one and onto then $C$ is congenial to $\mathcal{B}$.

2. If $M_{B} \cong M_{C}$ then there exists $\phi: M_{B} \rightarrow M_{C}$ which is natural.

3. Suppose that $\mathcal{B}$ is amenable and $\mathcal{B}$ is congenial to $C$. Let $\pi: \mathcal{M}_{\mathcal{B}} \rightarrow \mathcal{M}_{C}$ be a map. $C$ is amenable if and only if $\operatorname{Ker}(\pi)$ is an $R$-submodule of $M_{\mathcal{B}}$.

4. Does there exist an amenable basis $\mathcal{B}$ such that $\mathcal{B}$ is $A$-simple for all proper congeniality basis $\mathrm{A}$ ? If so, $\mathcal{B}$ is called simple and $\mathcal{M}_{\mathcal{B}}$ is called a simple basic module. Another way to say this is, if $\mathcal{M}_{\mathcal{B}}$ is a module and for every $\mathcal{B}, \mathcal{B}$ is congenial to $C$, if $C$ is amenable then $\mathcal{B}$ and $C$ are mutually congenial".

5. If $\mathrm{B}$ is amenable then $\operatorname{Ker} \phi$ is submodule of $\mathcal{M}_{\mathcal{B}}$ if and only if $C$ is amenable. 


\section{BibLIOGRAPHY}

[1] G. Abrams, P. Ara and M. Siles Molina, Leavitt path algebras. A Premimer and Handbook. Springer, in press.

[2] G. Abrams and G. Aranda Pino, The Leavitt path algebra of a graph, Journal of Algebra, 293(2), 319-334, 2005.

[3] G. Abrams and J. J, Simon, Isomorphisms between infinite matrix rings: a survey, Contemporary Mathematics, 259, 1-12, 2000.

[4] P. Ara and K. R.Goodearl, Leavitt path algebras of separated graphs. Journal fr die reine und angewandte Mathematik (Crelles Journal), 2012(669), 165-224.

[5] N. Bourbaki, Elements of Mathematics, Algebra I, Hermann, Paris, 1974.

[6] X.W. Chen, Irreducible representations of Leavitt path algebras. arXiv preprint arXiv:1108.3726, 2011.

[7] S. H. Friedberg, A. J. Insel and L. E. Spence, Linear Algebra, 3rd ed, Prentice Hall, 1999.

[8] C. Heil, A basis theory primer: expanded edition, Springer, 2010.

[9] T.W. Hungerford, Abstract Algebra: An Introduction. Saunders, Philadelphia, 1990.

[10] J. Lepowsky, and H. Li, Introduction to vertex operator algebras and their representations. Vol. 227. Springer, 2004.

[11] C. P. Milies and S. K. Sehgal, An introduction to group rings. Vol. 1. Springer, 2002.

[12] A. G. Paraskevopoulos, The Solution of Row-Finite Linear Systems with the Infinite Gauss-Jordan Elimination The Case of Linear Difference Equations with Variable Coefficients, arXiv preprint arXiv:1403.2624, 2014. 
[13] A. G. Paraskevopoulos, The Infinite Gauss-Jordan Elimination on Row-Finite $\omega \times \omega$ Matrices. arXiv preprint arXiv:1201.2950, 2012.

[14] M. Siles Molina, Algebras of quotients of path algebras. Journal of Algebra, 319(12), 5265-5278, 2007.

[15] S. Roman. Advanced linear algebra. Vol. 135. Springer, 2007. 


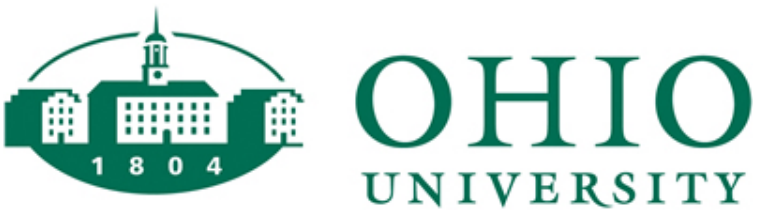

Thesis and Dissertation Services 